\title{
A hybrid branch-and-bound and Benders decomposition algorithm for network design problem
}

\author{
Saeed Asadi Bagloee, Majid Sarvi \\ Melbourne School of Engineering The University of Melbourne, Victoria 3010, Australia \\ $\&$ \\ Michael Patriksson \\ Chalmers University of Technology and University of Gothenburg, SE-412 96 Gothenburg, Sweden,
}

\begin{abstract}
Given a set of candidate road projects associated with costs, finding the best subset with respect to a limited budget is known as the Network Design Problem (NDP). The NDP is often cast in a bilevel programming problem which is known to be NP-hard (Ben-Ayed and Blair, 1990). In this study, we tackle a special case of the NDP where the decision variables are integers. A variety of exact solutions has been proposed for the discrete NDP, but due to the combinatorial complexity, the literature has yet to address the problem for largesize networks, and accounting for the multimodal and multiclass traffic flows. To this end, the bi-level problem is solved by Branch-and-Bound. At each node of the search tree, a valid lower bound based on system optimal (SO) traffic flow is calculated. The SO traffic flow is formulated as a mixed integer, non-linear programming (MINLP) problem for which the Benders decomposition method (Benders, 1962) is used. The algorithm is coded on a hybrid and synchronized platform consisting of MATLAB (optimization engine), EMME 3 (transport planning module), MS Access (database) and MS Excel (user interface). The proposed methodology is applied to three examples including Gao's network defined in (Gao et al., 2005), the Sioux Falls network (Farvaresh and Sepehri, 2013) and a real size network representing the city of Winnipeg, Canada (INRO, 2009). Numerical tests on the network of Winnipeg at various budget levels have shown promising results.
\end{abstract}

\section{INTRODUCTION}

Traffic congestion is a chronic challenge for cities. In addition to the demand management sometimes making wise investments into expanding the supply side is inevitable. Such investments have to be efficient, and this motivates formulating and solving a bilevel form of network design problem (NDP). The exposition of the NDP in the literature is as follows: There are a number of candidate road extension projects with associated costs and a limited budget in which not all costs are scaled. Hence the problem is postulated as finding the best choice of affordable candidate projects while accounting for the way the users (drivers) utilize the network. Such a premise is formulated as a bilevel programming problem (Magnanti and Wong, 1984) in which the total cost (travel time) incurred by the commuters is minimized. The problem is subject to the commuters' behaviour obeying the principles of User-Equilibrium (UE) which in itself is a programming problem known as the traffic assignment problem (TAP). It has already been established that the NDP is NP-hard (Balakrishnan et al., 1997; Magnanti and Wong, 1984); that is, as the network becomes bigger, the problem in general becomes computationally prohibitive.

The complexity of the NDP is rooted in two features: the bilevel and discrete nature of the problem. Any bilevel programming problem, even in its simplest configuration (i.e. objective functions and constraints being linear) is NP-hard (Ben-Ayed and Blair, 1990; Colson et al., 2005, 2007; Dempe, 2003). The decision variables are binary (1: to build; and 0: not to build (the project)) and the objective functions are non-linear which make the NDP a bilevel mixed integer non-linear programming (B-MINLP) problem. In the literature, the phrase "discrete network design problem (DNDP)" is used to emphasize the inclusion of binary elements (rather than continuous decision variables) into the general NDP. Interested readers in the continuous network

This is the author manuscript accepted for publication and has undergone full peer review but has not been through the copyediting, typesetting, pagination and proofreading process, which may lead to differences between this version and the Version of Record. Please cite this article as doi: 10.1111/mice.12224.

This article is protected by copyright. All rights reserved. 
design problem can consult with (Lin, 2011; Unnikrishnan and Lin, 2012; Waller et al., 2006).

Despite such complexity, the NDP in general and DNDP in particular have been studied extensively in many disciplines such as computer science, electrical engineering and mathematics. Thorough discussions of the problem can be found in (Farahani et al., 2013; Magnanti and Wong, 1984; Yang and Bell, 1998). The approaches taken in the literature can be classified as exact or heuristic. The exact methods aim to arrive at a global optimal solution but their applications to real networks are restricted. On the other hand, the heuristic methods aim to render good solutions for sizeable networks within an acceptable computational time by relaxing some crucial properties of the problem (such as discreetness of the decision variables) (Wong, 1985)

Furthermore in recent years, rapid expansions in the developing and emerging economies (largely in Asia and the Middle East) have made the DNDP relevant even amongst practitioners. According to the authors' exposures to the industry, in spite of the time-effectiveness of the heuristic methods, they are not appealing to the industries. It is mainly due to the fact that the NDP itself does not map well to the realities of transportation project selection, where the political and environmental considerations in generating a list of alternatives are paramount. This also is due in part to the fact that the heuristic methods provide supposedly good, but sometimes non-deterministic (or random) solutions. Such an uncertainty and the lack of stability of the results make it difficult to sell them to the (authorities) clients. Moreover, as long as the best solution is not known, the goodness degree of the solutions remains obscure.

Fortunately, ongoing enhancement in computational technology gives momentum to pursue exact methods (Wang et al., 2015). In one estimate, during the course of a decade, optimization had become a million times faster thanks to improvements in hardware as well as software (Lodi, 2010). On the other hand, although the size of the networks dealt with in the industry are large, the number of candidate projects (decision variables) is limited (say a dozen or so). It is the number of binary variables (and not the size of the networks) that significantly demarcates the solution spaces (Bagloee et al., 2013).

Given the above-mentioned characteristics of the problem and ayaiłable computational technology, we develop in this study an exact method for the DNDP, tailored to real-size networks. Throughout this study, we assume that travel demand is fixed, deterministic and exogenous. The methodology proposed here includes two important features of the real networks that have been largely neglected in the literature: (i) multimodal: consideration of private traffic flow as well as public or transit flow and (ii) multiclass: various distinct classes of private traffic flow including cars, trucks, and HOV, etc. To the best of our knowledge, no such attempt (employing exact methods for real networks subject to multimodal and multiclass traffic flow) has been made before ${ }^{1}$.

In order to solve the problem, an efficient branchand-bound $(\mathrm{B} \& \mathrm{~B})$ algorithm hybridized by the Benders decomposition method (dubbed as B\&BB) has been devised. The B\&B method is employed to address the discreteness nature of the DNDP (Boyce et al., 1973; Chen and Alfa, 1991; Leblanc, 1975), and the Benders decomposition method is used to find tight lower bounds at the nodes of the $\mathrm{B} \& \mathrm{~B}$ tree. The hybridization refers to the fact that the Benders decomposition method is used to calculate a lower bound for a newlygenerated node in the tree.

In the quest to tailor the methodology for largesize networks, a variety of innovative techniques are developed. Node selection and the branching rules of $\mathrm{B} \& \mathrm{~B}$ are made based on a merit index computed for each of the candidate projects. We also add a term called budget consumption to tap into the fact that projects are wisely chosen. Hence the optimum solution is intuitively supposed to utilize the allocated budget as much and effectively as possible. We also add alpha (varies between 0 and 1), a term devised to speed up the algorithm when dealing with large-size networks. It is embedded in the lower bound calculations as the search on B\&B proceeds, which is specified upon the employed computational technology. We also developed a memoryless search mechanism over $\mathrm{B} \& \mathrm{~B}$; that is, the algorithm does not need to remember the entirety of the tree which rapidly expands and makes the RAM a serious cause of concern.

The objective function is the total travel time spent in the network. The algorithm is launched by an intuitively good solution for which the multimodal, multiclass UE traffic assignment problem (MMMC-UE-TAP) is computed. It is worth noting that the sub-problem MMMC-UETAP can also be replaced with any other traffic assignment models such as quasi-dynamic,

\footnotetext{
${ }^{1}$ Although some scholars may prefer a simpler and more parsimonious model based on single class traffic flow, this research appeals to the industry as well. The first author was recently involved in a mega network design and prioritization project in the Middle East. The client rejected any thought other than a try-error approach on its multimodal multiclass traffic assignment model simply because what were proposed - though derived from a sophisticated methodology - lacked multimodal, multiclass aspects. Of course, these aspects add to the complexities of calculations, but it is the cost we incurred to entice practitioners in the industry. Nonetheless, it did not preclude us comparing our findings with what is reported in the literature for networks of Gao and Sioux Falls. It was a matter of switching off the "multi" to turn everything to a single-class model.
} 
dynamic or stochastic etc. Nonetheless, the UE principles are widely used and recognized among scholars due to their widespread applications in research as well as practice.

In the remainder of the article, Section 2 contains a literature review; Section 3 provides the general formulation of the problem; in Section 4, the Branch and Bound algorithm is introduced; Section 5 is dedicated to the Benders decomposition method; numerical results are provided in Section 6; and finally these are all followed by the conclusions in Section $7^{2}$.

\section{LITERATURE REVIEW}

A thorough discussion on general NDP can be found in (Farahani et al., 2013; Magnanti and Wong, 1984; Minoux, 1989; Yang and Bell, 1998). In this section, we first provide a synthesized overview of the literature with a primary focus on the exact methods. We then elaborate on recent evolutions in the literature over the course of the last two decades.

Among the pioneers, Leblanc (1975) solved the DNDP using a branch-and-bound (B\&B) algorithm. Poorzahedy and Turnquist (1982) approximated the total travel time function in the upper level to a well behaved function and arrived at a single-level formulation that was then solved by a heuristic B\&B algorithm. Farvaresh and Sepehri (2013) proposed a more efficient B\&B. Generally speaking, the difficulty in applying the $\mathrm{B} \& \mathrm{~B}$ method lies in how to adapt the methodology to deal with the intrinsic

\footnotetext{
${ }^{2}$ Some believe that the network design problem (NDP) is on old topic which refuses to die a dignified death and practitioners have considered and rejected this mathematical oddity. In addressing such concerns, there are three points worth noting. (i) In light of our exposure to the real projects in the industry (some of us have a long standing career in the international consultants), we first acknowledged that there is a deep gap between academic and real engineering, while the problem is becoming more relevant. The main problem is that it is hardly to find a paper tailoring methodology for large-size networks. In addition, cities' traffic models are more complicated than benchmark networks such as Sioux-Falls. For instance, multiclass and multimodal features are indispensable parts of such models, which are largely simplified in the literature. So we in the university have yet to offer a product meeting real needs of the industry. (ii) Problems such as network design problem (NDP) deserved to be viewed as milestone or benchmark problems which test our knowledge and computational technologies at time. Working toward such milestone problem may bring some other advantageous byproducts. For instance, in the quest to solve the discrete NDP, we found out that the lagrangian sub-problem (of the Benders decomposition method) is in fact a capacitated traffic assignment which is still a live subject. Furthermore, the algorithm developed for the NDP can also be applied to other descending problems such as congestion pricing, facility placement, transit priority lane design etc. (iii) The types of NDP interest scholars in operational research especially when mixed integer problems (MIP) are of interest. The MIP has widespread applications in management science, manufacturing designs, decision making and planning etc.
}

non-convexity of the DNDP due to the implicit, non-linear UE constraints (Wang et al., 2013). (Gao et al., 2005) introduced the concept of support function to include new additional projects into the traffic flow by which the bilevel DNDP was transformed into a general, mixed, non-linear problem. They then employed the Generalized Benders Decomposition method as a solution algorithm. Wang and Lo (2010) employed singlelevel, mixed-integer linear programming (MILP) to approximate a continuous network design problem (CNDP) that dealt with the continuous expansion of existing links. The non-linearity of the travel time function was removed by applying a convexcombination based piecewise linear approximation. Wang and Lo (2010) employed complementary constraints for UE traffic flow to arrive at a singlelevel problem for which a convex-combination based piecewise linear approximation was developed as a solution algorithm. Luathep et al. (2011) transformed the DNDP to a single-level problem in which variational inequality (VI) constraints represent the UE conditions, followed by a cutting plane based algorithm to seek the optimal solution. (Farvaresh and Sepehri, 2011) replaced the UE conditions with equivalent Karush-Kuhn-Tucker (KKT) conditions which led to a single-level, mixed-integer linear problem. (Wang et al., 2013) expanded the NDP model to find the optimal number of lanes to existing candidate roads. Fontaine and Minner (2014) employed a piecewise linear approximation scheme to arrive at a single-level, mixed integer linear problem to be solved by Benders decomposition method. There are also heuristic approaches for the DNDP in the literature in which a variety of methods such as genetic algorithm, ant system and hybrid meta-heuristics are used. A thorough review of these methods is provided by (Bagloee et al., 2013))

As the above review shows, a general approach to bilevel programming problems such as the considered NDP is to transform the problem into a single-level problem (Colson et al., 2005, 2007; Dempe, 2003). The convention is to replace the lower level decisions by an implicitly-determined function (reaction function) or by its Karush-KuhnTucker (KKT) conditions. The resulting singlelevel MINLP problem is then solved by various methods such as Benders decomposition, Lagrangian relaxation, descent methods (such as sequential quadratic programming), outer approximation, branch-and-bound, penalty function methods, and trust-region methods (Floudas, 1995; Leyffer, 1993; Li and Sun, 2006).

Gao et al. (2005) developed a methodology based on the concept of support function to transform the bilevel NDP problem into a single-level MINLP. The resulting problem is then solved using Generalized Benders Decomposition (GBD) 
algorithm (Geoffrion, 1972). It is numerically shown that their method fails to find a global optimum solution in some cases (Farvaresh and Sepehri, 2013).

Zhang and Gao (2009) formulated a mixed, continuous and discrete NDP as a single-level mathematical program with complementarity constraints to represent the UE traffic flow. Although the numerical results presented for smallscale examples are promising due to employing a locally convergent algorithm, the capacity of the methodology to arrive at optimal solutions has yet to be investigated.

Wang and Lo (2010) developed a single-level optimization formulation with complementary constraints for UE traffic flow that turn the DNDP into a mixed integer linear programming (MILP) problem. The resulting MILP model is based on enumerating the paths between origin-destination (OD) and a piecewise linear approximation of the link travel time functions with binary decision variables. Thus, the outcomes are significantly dependent upon the linearization scheme. With respect to the path enumeration component, its application to sizable networks has yet to be investigated.

Similarly, Luathep et al. (2011) formulated the DNDP as a single-level optimization problem with a Variational Inequality (VI) constraint representing the UE conditions. The VI constraint efficiently obviates the need for path enumeration based on the accumulated number of extreme points. Nonetheless, the results are significantly dependent upon the linearization scheme made on delay functions. The network of Sioux-Falls is used for numerical analysis. In view of the number of extreme points used for representing the UE condition, application to large scale networks has yet to be investigated.

Farvaresh and Sepehri (2011) developed a singlelevel MILP by representing the UE conditions as KKT conditions and employing linearization schemes. To do so, the non-linear delay function is replaced (or approximated) by some linear segments. Hence the linearization scheme refers to the location and number of segments. The final results vary over different linearization parameters.

Farvaresh and Sepehri (2013) address the bi-level aspect of the problem explicitly by developing a Branch-and-Bound algorithm based on the seminal work of Leblanc (1975) while a more efficient lower bound is sought. Given a feasible binary solution, the UE Traffic Assignment Problem (UETAP) is solved to obtain an upper bound of the total travel time. A valid lower bound is also obtained by solving a system optimal (SO) version of the NDP. Due to the usually significant gap between these bounds (Roughgarden and Tardos, 2002), the applications are limited to special cases in which the difference in traffic flow under UE and SO conditions is negligible.

Wang et al. (2013) recently expanded the NDP to consider a number of additional lanes as decision variables. They first relaxed the bilevel programming model by formulating a single-level problem in which an SO (not UE) network design problem is solved. Two methods based on the relationship between UE and SO principles are developed. The first method, termed SO relaxation, takes advantage of the property that an optimal network design decision under SO traffic flow condition can be regarded as an approximate solution with UE traffic flow. The second method, termed UE reduction, reduces the gap between the bilevel programming model and the single-level model by adding convex inequalities based on the UE model's (objective) function (Beckmann et al., 1956) to the constraints of the relaxed problem. Similar to the work of Farvaresh and Sepehri (2013), the efficiency of the proposed methodology hinges on the assumption that the UE and SO solutions are similar which is not the case in real networks (Roughgarden and Tardos, 2002).

Fontaine and Minner (2014) developed a scheme by which the upper-level objective function as well as the objective function of the lower-level problem are approximated by piecewise linear functions as well as the Beckmann function of the UE traffic flow. The bi-level problem is then transformed into a single-level problem by representing the UE conditions through its corresponding KKT conditions. Benders decomposition is then employed to solve the resulting problem. Similarly, the quality of the solution as well as the efficacy of the methodology is highly dependent upon the linearization scheme. Furthermore, a linearization of the Beckmann function compromises arriving at a global optimum solution. Numerical results for a medium sized example with 36 zones are reported.

As can be seen even among the most recent studies, no attempt has been made toward addressing large scale networks or considering multiclass and multimodal traffic flow. Furthermore, a clear majority of the algorithms utilize simple approximations of the upper and/or lower-level objective functions. The conventional wisdom in the literature is to cut away the intractable organs of the problem in the first place with a view to arriving at a more benign and wellbehaved problem (Poorzahedy and Turnquist, 1982). Such approaches may deprive the problem from the dimensions based on which the DNDP stands, to the extent that the solutions become overly simplified and unreliable whereas in this study, we aim to lay down a solid exact foundation embracing the DNDP in its full capacity.

The next section provides a mathematical formulation of the DNDP subject to multimodal and multiclass traffic flow. 


\section{FORMULATION OF THE DNDP}

Define:

$A, A^{\prime}$ : Sets of existing roads (or arcs), and candidate road projects (projects in short), respectively

$N$ : set of nodes

$B$ : budget

$y_{a}$ : binary decisions variable of project $a \in A^{\prime} ; 1$ :

to build and $0:$ no to build

$c_{a}$ : construction cost of project $a \in A^{\prime}$

$x_{a}, \bar{x}_{a}$ : auto and transit traffic flows (both in

passenger car unit-PCU) on link $a \in A \cup A^{\prime}$

respectively

$t_{a}\left(x_{a}+\bar{x}_{a}\right):$ travel cost or time or delay of link

$a \in A \cup A^{\prime}$, defined by a non-decreasing BPR

function (Spiess, 1990) of link flow $x_{a}+\bar{x}_{a}$ (called

delay function). Some studies have illustrated the

highly nonlinear nature of the travel time (Lo et al., 2006). Nonetheless the delay functions can be of any forms other than BPR as long as they are nondecreasing and differentiable.

$A_{n}^{-}, A_{n}^{+}$: set of links starting and ending at node

$n \in N$ respectively; $A_{n}^{-}, A_{n}^{+} \subset A \cup A^{\prime}$

$M$ : set of distinct user classes

$b_{a}^{m}$ : additional delay (constant bias) perceived by auto class $m \in M$

$x_{a}^{m}$ : traffic volume of auto class $m \in M$ of link $a \in A \cup A^{\prime}$, in other words: $x_{a}=\sum_{m \in M} x_{a}^{m}$ (see

Equation (7)

$O, D, Q$ : set of origins, destinations and origindestination pairs respectively, $Q=O \times D$.

$q_{i}, \bar{q}_{i}$ : auto and transit travel demand in PCU for origin-destination $i \in I$ respectively.

$P_{i}$ : set of paths between origin-destination, $i \in I$.

$h_{k}, h_{k}^{m}$ : Total flow of all auto classes and flow

pertaining to class $m \in M$ on path $k \in P_{i}$,

respectively: $h_{k}=\sum_{m \in M} h_{k}^{m}$ (combination of all

traffic flow of different classes constitutes total volume on path $k$ )

$\delta_{a, p}^{m}$ : link-path incident index, 1 if link $a \in A \cup A^{\prime}$

belongs to path $p$ pertaining to class $m \in M$ and 0 otherwise

$\bar{h}_{k}$ : transit flow in PCU on path $k \in P_{i}$

$\bar{\delta}_{a, p}$ : it is 1 if link $a \in A \cup A^{\prime}$ belongs to path $p$

pertaining to transit network

$w_{n}$ : average waiting time at node $n \in N$ pertaining

to transit system

$f_{a}$ : sum of frequency of service for all transit lines

on link $a \in A \cup A^{\prime}$

$U$ : is a sufficiently large value, total demand

$\sum_{i}\left(q_{i}+\bar{q}_{i}\right)$.
The bilevel DNDP may be written as follows:

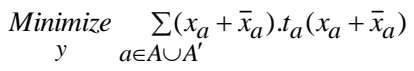

(1)

subject to

$$
\begin{array}{ll}
\sum_{a \in A^{\prime}} c_{a} \cdot y_{a} \leq B, & a \in A^{\prime}, \\
y_{a} \in\{0,1\}, &
\end{array}
$$

(3)

$\underset{x, \bar{x}}{\text { Minimize }} \sum_{a \in A \cup A^{\prime}} \int_{0}^{x_{a}} t_{a}\left(x_{a}+\bar{x}_{a}\right) d x+\sum_{m \in M} \sum_{a \in A \cup A^{\prime}} x_{a}^{m} \cdot b_{a}^{m}$,

(4)

subject to

$\sum_{m \in M} \sum_{k \in P_{i}} h_{k}^{m}=q_{i}, \quad i \in Q$,

(5)

$$
x_{a}^{m}=\sum_{i \in I} \sum_{k \in P_{i}} h_{k}^{m} \delta_{a, p}^{m}, \quad a \in A \cup A^{\prime}, \forall m \in M,
$$

$$
x_{a}=\sum_{m \in M} x_{a}^{m}, \quad a \in A \cup A^{\prime}, \forall m \in M,
$$

(7)

$$
x_{a} \leq U \cdot y_{a}, \quad a \in A^{\prime},
$$

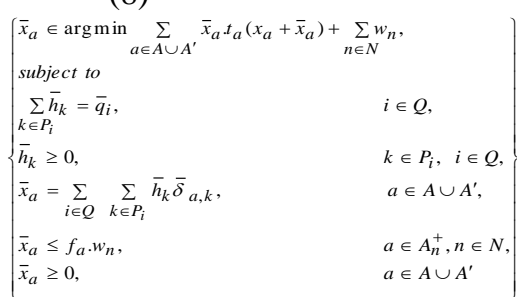

(9)

$x_{a}^{m} \geq 0$,

$a \in A \cup A^{\prime}, m \in M$

(10)

Equation (1) describes the upper-level goal of minimizing the total travel time. Mathematical expressions (2) and (3) ensure the feasibility of the projects with respect to their construction costs and available budget. At the lower level, (mathematical expressions (4) - (7)) the Beckmann formulation of UE flow consists of $m$ distinct auto classes is computed. Constraint (8) ensures that projects corresponding to no-build decisions $\left(y_{a}=0\right)$ are excluded from the traffic assignment ( $U$ is a sufficiently large value, total demand $\sum_{i}\left(q_{i}+\bar{q}_{i}\right)$ Equation (9) carries out transit assignment based on optimal strategy (Spiess, 1993; Spiess and Florian, 1989)) and it returns $\bar{x}_{a}$ as additional or background traffic volume in PCU to be considered in the traffic assignment. The multiclass facet of the traffic assignment is embedded in the interpretation of the bias term $b_{a}^{m}$ (pertaining to vehicle class and link $a$ ) in which all distinct auto classes using link $a$ are subject to a same congestion level (based on the total traffic volume of all class $t_{a}\left(x_{a}\right)$ plus an additional term (the bias term) exclusive to each 
class (i.e. $\left.t_{a}^{m}\left(x_{a}\right)=t_{a}\left(x_{a}\right)+b_{a}^{m}\right)^{3}$. Such formulations define a simplified way to consider the multiclass aspect of traffic flow ${ }^{4}$. The roads' delay functions are calibrated based on traffic survey data for which the bias term is the intersect value of the non-linear regression. In real practice for cities with traffic analysis models, the delay functions are already calibrated and provided.

In the following exposition we show that it is still a reliable alternative given the complexity of the other approaches.

\subsection{Treatment of Multiclass and Multimodal}

A comprehensive consideration of multiclass UETAP leads to an asymmetric and non-monotone user equilibrium model. For multiclass UE-TAP, a variety of methods such as Variational Inequality, Complementarity Method, Fixed-Points and Entropy Maximization has been proposed (Aashtiani, 1979; Bar-Gera and Boyce, 1999; Chen et al., 2011; Dafermos, 1972; Florian and Morosan, 2014; Nagurney, 2000; Nagurney and Dong, 2002; Zhang and Chen, 2010). Nevertheless, the literature has yet to come to a consensus on how to address the multiclass UE-TAP which is still the subject of ongoing debate (Boyee, 2014).

It is worth noting that the evolving knowledge in the state-of-the-art bush-based and origin-based algorithms such as algorithm B and TAPAS present a precious means of considering the multiclass feature as well. A recent review of the latest advances in the solution algorithms of the UE-TAP is provided by (Xie and Xie, 2014, 2015). Since these algorithms decompose the UE-TAP to the origins (i.e one-origin to all-destinations), one can

\footnotetext{
${ }^{3}$ It is worth noting that the Beckmann formulation is convex w.r.t to $x_{a}$, For each class, the shortest path computations of each class take into account the class-specific bias as well as the travel time given by the volume-delay. Therefore it is not necessary to store the class specific volumes explicitly $\left(x_{a}^{m}\right)$ the total volumes are sufficient $\left(x_{a}\right)$ Spiess, H., 1984. Contributions à la théorie et aux outils de planification des réseaux de transport urbain, $P h D$ dissertation. Montréal: Université de Montréal, Centre de recherche sur les transports, Université de Montréal. Centre de recherche sur les transports. INRO, 2009. EMME3 v 3.2, EMME3 User's Guide, 3.2 ed, Montreal, Quebec, Canada. ${ }^{4}$ The way that a multimodal traffic assignment is conducted in EMME is as follows: based on the headway and transit demand a prior estimation for the transit volume is made (i.e. $\bar{x}_{a}$ ). This

$\bar{x}_{a}$ is then treated as a background volume for the auto traffic assignment followed by conducting a transit assignment to get a more precise assignment result. Accordingly $\bar{x}_{a}$ in Eq (4) is treated as a constant term derived from transit assignment $(\mathrm{Eq}(9))$. Since our primary intention was to make use of a commercial software for the traffic assignment (i.e. EMME 3) we referred interested reader to the software's manual INRO, 2009. EMME3 v 3.2, EMME3 User's Guide, 3.2 ed, Montreal, Quebec, Canada.. and Boyce, D., 2014. Network equilibrium models for urban transport, in: Fischer, M.M., Nijkamp, P. (Eds.), Handbook of Regional Science. Springer Berlin Heidelberg, pp. 759-786.
}

further decompose the UE-TAP at each origin to the number of vehicle classes. Nevertheless in theory, the computational effort to solve the multiclass UE-TAP is multiplied by a factor of the number of distinct classes. Usually, practitioners deal with a relatively large number of classes (say a dozen), hence the computation time becomes a significant concern. Alternatively, we adopt the bias term (Spiess, 1984) to turn any multiclass case (no matter how many vehicle classes are involved) into a single-class TAP.

Similarly, the combination of (private) traffic and transit assignment (multimodal) results in a nonconvex programming problem for which uniqueness and stability of the solutions with respect to the inputs are not guaranteed (Florian and Morosan, 2014). The relevant studies either fail to fully consider the simultaneous interaction of private and transit modes, or suffer from high computation time (De Cea et al., 2005; Liu and Meng, 2012).

Given the complexities involved as described, the above formulation (Equations (4) - (9)) is empirically proven to be acceptable to addressing the MMMCUE-TAP (Spiess, 1984) such that it has been adopted in some transport planning software (INRO, 2009). In this study Equations (4) - (9) have been coded as a module in EMME 3 (INRO, 2009) and is summoned when needed.

In the next section we elaborate on the methodology developed to solve the DNDP.

\section{BRANCH-AND-BOUND} ALGORITHM

\subsection{Overview}

The bilevel DNDP expressed in Equations (1)-(9) is a mixed integer programming problem with $\left|A^{\prime}\right|$ set of binary decision variables. The discreteness of the problem is laid over a tree-shaped structure where each node of the tree represents a sub-area of the solution space, delineated by a sub-problem. The algorithm is first launched with a feasible solution for which the MMMC-UE-TAP is solved, and the corresponding objective function value is labelled as the incumbent value (as an iterative algorithm proceeds, the best solution found is labelled the "incumbent solution" and the corresponding objective value is called the incumbent value. In other words, the incumbent value is the least total travel time found as the algorithm proceeds through the iterations). Since the problem is of a minimization nature, the incumbent value is an upper bound value denoted by $U B^{*}$. The algorithm can be initiated with any feasible solution; perhaps the obvious one is the "do-nothing" scenario in which all binary variables are set to zero. Nevertheless, one can seek a more informed initial solution rather than the simple donothing, hoping that it facilitates the rest of the algorithm's process. 
A tree is then planted upon a root node representing the entire solution space. Once a new node is generated, a local lower bound is also calculated and tagged on the respective node. In case the lower bound is found to be above the incumbent value, the corresponding node is frozen (or fathomed) and consequently the respective unexplored part of the tree is discarded from further exploration since a better solution (i.e. the incumbent value) has already been secured. As a result, it is very desirable to arrive at fathoming cases (lower bound $>$ incumbent value) to cut the solution spaces as much as possible, otherwise the algorithm chooses an unfathomed node and branches out two new nodes (in other words, the respective solution space is further split into two smaller sub-areas). This process of finding no unfathomed node, a lower bound calculation and a comparison with the incumbent value will proceed until an unfathomed node is found. In this quest as the tree grows, the sub-areas represented by nodes deep down the tree become smaller and smaller. Sometimes, a sub-area or a sub-problem (represented by a node of the tree) becomes too small, in such a way that it contains only one feasible solution. Whenever the tree reaches a feasible solution, the corresponding MMMC-UETAP is solved and accordingly the incumbent value is updated. At the end, the incumbent value and the corresponding feasible solution is the final solution.

The questions remaining to be answered are as follows: (1) Node selection: which node must be chosen for branching? (2) Branching rule: once a node is chosen, how to split the sub-area? And (3) Lower bound: how to calculate the lower bounds?

We discuss the first two questions in the next subsection as navigation on the tree. We then elaborate on the lower bound in the next section.

\subsection{Navigating in the tree}

Each node in the tree represents either a sub-area or a feasible solution. A sub-area is encoded as a string of binary values (" 1 " and " 0 ") to indicate whether the respective project is to be constructed or not constructed, and " 2 " is yet to be decided (either 1 or 0 ). For instance, the string "01022" depicts a sub-area consisting of five candidate projects where the first three components are to be build/no-build (0/1) and the last two (represented by "2") are unspecified (yet to be decided). In other words, subarea "01022" encompasses all feasible solutions in which the first three projects are fixed as " 010 " and the last two are either of the following combinations: " 00 ", " 01 ", " 11 ”, or " 10 ".

As the tree grows, at each iteration, a node " $z$ " representing a sub-area needs to be chosen for further branching. To this end, an undecided project (a project represented by value "2") has to be selected to be replaced by 0 (no-build) and 1 (tobuild) on two new branches. The result is that it will create two new nodes.
During this mitotic phase in which the tree continues to expand, the algorithm is constantly faced with the decision of which node with which partial solution to choose for branching. Once a node is selected, the algorithm must still pick one undecided project of the corresponding partial solution to complete the next phase of branching. To this end, there are some methods that require additional problem solving or the retrieval of the entire database with a view to finding the best node for branching. As the size of the network increases, such methods become computationally intensive.

\subsubsection{Branching rule based on merit index:}

Alternatively, via a novel approach, we consider the order of the projects placed in the string from left to right as priority for node selection and branching. Therefore, a merit index is first defined and calculated for each project. The projects are then sorted from the highest to the lowest to be placed accordingly in the string.

The merit index aims to find the most likely projects among the candidates. As such, the merit index is defined as following the mathematical expression $x_{a} / v_{a} / c_{a}$ where $v_{a}$ stands for capacity of road $a \in A^{\prime} 5$. The rationale behind the proposed merit index is based on two considerations: (i) the traffic volume alone is not a good enough indicator to prompt us to increase the qualification chance of a candidate; the capacity also needs to be considered. The more congested a road (i.e., higher volume-per-capacity $x_{a} / v_{a}$ ), the more demanding the road is and hence it may deserve to be put forward for the construction; and (ii) between two roads with similar traffic conditions, it is a wise choice to choose the one that would result in the lowest construction cost; therefore the volume-percapacity in the merit index is normalized by the construction cost.

Consequently, the branching rule becomes very simple: first sort the projects based on their merit indices in descending order. For branching, there is only one rule: choose the very next undecided project in the corresponding string.

\subsubsection{Node Selection Rule:}

As for node selection, the algorithm abides by two simple rules: (i) choose the deepest node of the tree; and (ii) in the case of two nodes at the same level, choose the one located on a branch associated with $y_{a}=1$.

There are two advantages with such a convention: (i) given the fact that the projects are sorted on a merit basis, it makes sense to go deep into the tree to select the next best project for branching in such a "greedy" manner that would aim for finding the optimum solution lying there; and (ii) the algorithm need not save/retrieve/process the information of

\footnotetext{
${ }^{5}$ The merit index is calculated based on the scenario in which all the projects exist.
} 
the entire tree. Once a new node is made, it is yet to be further processed for lower bound/fathoming. At each node, the algorithm just needs to move forward as much as possible on the paths that consist of $y_{a}=1$ branches. In case there is no space for such moyement, the algorithm moves only one node back to the previous node and then moves through the $y_{a}=0$ and then follows $y_{a}=1$ branch (if possible). This process carries on until the termination criterion is met. Figure 1 illustrates the gradual build-up of the tree based on these rules.

The algorithm does not need to remember the already traversed paths nor the paths ahead as shown in Figure 1 as the structure expands; it just needs to know the lower bounds of the nodes on the current path plus the best solution found so far which is a string of binary values $(0 / 1)$ and the corresponding incumbent value (we call it a memoryless search mechanism). For example, if the current node is (11002), the next move is to process node (11001) followed by the node represented by (11000). For the third move, the algorithm moves three nodes back to reach node (10222).

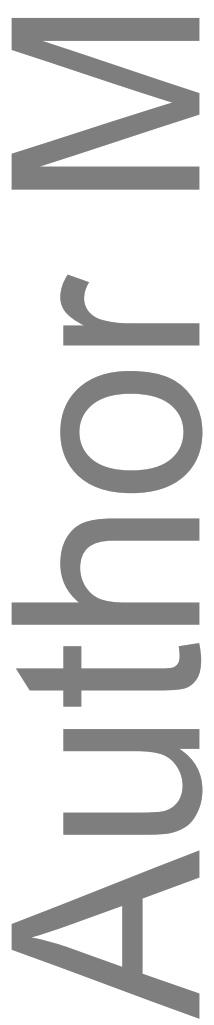

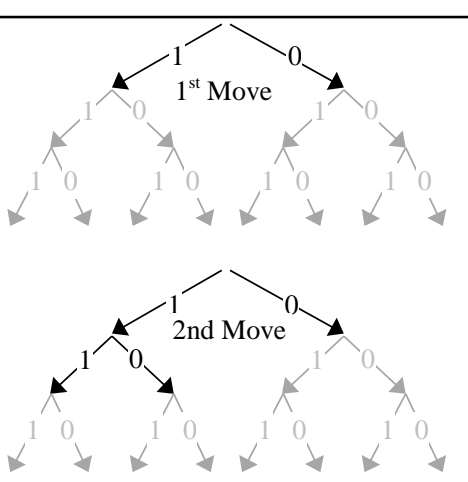
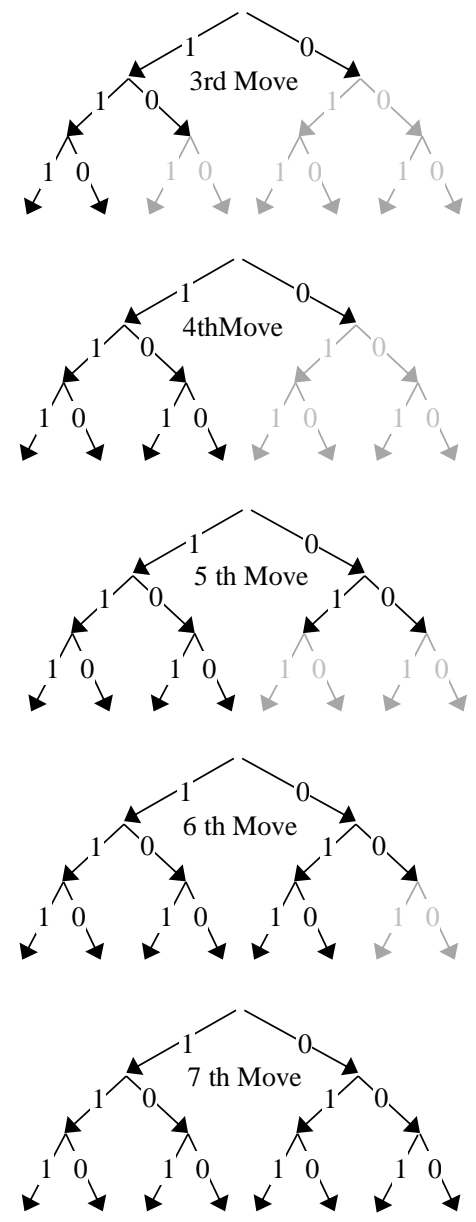

- Starting solution at root node: "222"

- Node selection rules: (i) choose the deepest node of the tree (ii) in case of having two nodes at the same level choose the one that was made of a branch representing $y_{a}=1$.

- Branching rule: choose the very next undecided project in the

Figure 1 Proposed node selection and branching in the branch-and-bound algorithm

\section{LOWER BOUND}

One of the primary concerns in any B\&B is the method of calculating lower bounds at the tree's nodes. For the sub-area represented by "01022", it is necessary to compute a lower bound on the objective function of (equation (1)) evaluated at all 
possible and feasible combinations: "01000", "01001", “01010", and "01011". An apparent method of computing a valid lower bound is to set all undecided variables equal to one and solving the MMMC-UE-TAP which results in the UE flows on the resulting network. According to Braess Paradox (Braess, 1968; Braess et al., 2005), such measure may result in worse-off traffic or a higher lowerbound. To this end, replacing the User Equilibrium flow with System Optimal traffic flow (MMMCSO-TAP) ensures valid and decreasing successive lower bounds (Leblanc, 1975). Nevertheless in a congested network, there is a deep gap between UE flow and SO flow which leads to a very loose lower bound. This means that it is unlikely to truncate the solution space due to arriving at lower bounds above the incumbent value (upper bound).

In such cases, the algorithm has to process every feasible solution and as a result, the algorithm will have no superiority over an exhaustive enumeration. In fact, it becomes much worse because in addition to calculating the upper bounds as required in the enumeration, the algorithm has to calculate the lower bounds as well.

In order to obtain a tighter lower bound once a new node is added to the tree, one can seek a solution out of the following problem (Leblanc, 1975):

$$
\min \sum_{a \in A \cup A^{\prime}} x_{a}^{m} t_{a}^{m}\left(x_{a}\right) \text { s.t }(2),(3),(5),(6),(7),(8) \text { and }
$$

$(10)^{6}(11)$

In optimization problem (11), the non-linear convex objective function is subject to some linear constraints and the outputs consist of binary decision variables $\left(y_{a}\right)$ as well as traffic flows which are continuous variables $\left(x_{a}\right)$. Hence, it is a Mixed Integer Non-Linear Programming (MINLP) for which we develop a solution algorithm based on the Benders deeomposition method (Benders, 1962; Lasdon, 2013). In fact, the lower bound formulated in (11) is a system-optimal discrete network design problem (SO-DNDP). In some studies, the SODNDP itself is treated as an approximation approach to address the DNDP (while we employ it as a lower bound). The beauty of such formulation rests in the fact that the bi-level DNDP is easily dissolved into a single-level problem which is much more easily solved.

\subsection{Benders Decomposition Method For}

\section{MNLP}

Consider the original problem (11) in the general ${ }^{7}$ form of MINLP as follows:

\footnotetext{
${ }^{6}$ As discussed before, the output of the transit assignment is $\bar{x}_{a}$ which is viewed as additional or background traffic volume to be considered in the UE traffic assignment. Therefore the travel time function can be first updated to accommodate the $\bar{x}_{a}$ before carrying out the traffic assignment. Hence the notation $\bar{x}_{a}$ as well as equation (9) can be omitted from the formulation. Furthermore
}

$O P: \underset{x, y}{\operatorname{Min}} f(x, y)$ s.t. $S(x, y) \leq 0, x \in X \subset \mathfrak{R}^{n}, y \in Y=\{0,1\}^{q}$

(12)

where $f$ is the objective function $\left(\sum_{a \in A \cup A^{\prime}} x_{a} t_{a}\left(x_{a}\right)\right)$, $x$ is the vector of links flows, $y$ is the vector of binary decision variables along with set $S$ delineate solution space to which the problem is subject. Consider eliminating the binary decision variables by fixing them to some feasible values $\left(y^{i} ; i\right.$ is iteration counter), hence the problem changes to searching over feasible $x$. We refer to it as the "Primal Sub-Problem (PSP)":

$$
P S P(i): \operatorname{Min}_{x} f\left(x, y^{i}\right) \text { s.t. } S\left(x, y^{i}\right) \leq 0, x \in X \subset \mathfrak{R}^{n}
$$

Once it is solved (if it exists), the corresponding traffic volume $x^{i}$ and Lagrange multipliers of the constraints $\omega^{i}$ are obtained ${ }^{8}$. The partial dual (Lagrange) format of the objective function can be written as $L\left(x^{i}, y, \omega^{i}\right)=f\left(x^{i}, y\right)+\omega^{i} . S\left(x^{i}, y\right)$. According to "weak duality theorem", each feasible solution (y) to the dual problem is a lower bound to the original problem. Therefore, given $\left(x^{i}, \omega^{i}\right)$, the algorithm seeks a new set of feasible binary variables for the next iteration $\left(y^{i+1}\right)$ by solving the following problem which is called "Relaxed Master Problem (RMP)":

$$
R M P(i): \operatorname{Min}_{y \in \bar{Y}, V} V \text { s.t. } V \geq \min L\left(x^{k}, y, \omega^{k}\right) ; k=1 . . i
$$

The problem (14) is a Mixed Integer Linear Programming (MILP) problem which is easier to solve (compared to MINLP), even for sizable problems. The right hand side of the constraint is a succinct way of representing a series of linear constraints to which " $\mathrm{v}$ " must be found greater than the minimum of them. Note that $y \in \bar{Y}$ ensures feasibility of the binary variables where $\bar{Y}$ is feasibility observing two points: (i) it entails all combinations of binary variables that satisfy the budget constraint $(\mathrm{Eq}(3))$; and (ii) the linear constraints in the relaxed problem are actually viewed as Benders cuts (cutting planes) to the solution space collected from the first iteration to current iteration $i$, (i.e. $k=1 . . i$ ). In order to obtain

\footnotetext{
${ }^{7}$ Note that $x, y$ denote continuous traffic volumes and binary decision variables. In analogous to problem (11) consider $S(x, y) \leq 0$ represents constraints $(2),(3),(5),(6),(7),(8)$ and (10) and $f(x, y)=\sum_{a \in A \cup A^{\prime}} x_{a}^{m} t_{a}^{m}\left(x_{a}\right)$.

${ }^{8}$ In order to solve the PSP and finding the traffic volumes and the lagrangian values we have developed a method known as Inflated Travel Time Bagloee, S.A., Sarvi, M., 2015a. Heuristic Approach to Capacitated Traffic Assignment Problem for LargeScale Transport Networks. Transportation Research Record: Journal of the Transportation Research Board, 1-11. which is discussed in section 5.2.1.
} 
an always new $y^{i+1}$ at the current iteration $i$, the solutions that fail to satisfy the following constraints are discarded from $\bar{Y}$ (Balas and Jeroslow, 1972)).

$$
\sum_{a \in Y 1} y_{a}-\sum_{a \in Y 0} y_{a} \leq|Y 1|-1, Y 1=\left\{a \mid y_{a}^{k}=1\right\} ; Y 0=\left\{a \mid y_{a}^{k}=0\right\},
$$

where $Y 1, Y 0$ represents the candidate projects that have taken value of 1 and 0 respectively in the respective solution $k$.

On the one hand, the result of the primal subproblem $P S P(i)$ is a feasible solution, so the value of $f\left(x, y^{i}\right)$ out of the PSP(i) is an upper bound on the optimal value of the original problem. On the other hand, as mentioned before, the relaxed master problem is in fact a relaxed dual problem to the original problem, hence $v$ out of the $R M P(i)$ is a lower bound on the original problem's optimal value. Consequently, the solution algorithm is set out as solving primal and relaxed problems $(P S P(i), R M P(i))$ alternatively until the lower and upper bounds get within a close enough proximity to each other

\subsection{Benders Decomposition Formulation For} System Optimal MINLP

Let us rewrite the original problem given in formulation (11) by expanding on the traffic volumes:

$$
\begin{aligned}
& O P \min \sum_{a \in A} x_{a} \cdot t_{a}\left(x_{a}\right)+\sum_{a \in A^{\prime}} x_{a} \cdot t_{a}\left(x_{a}\right)+\sum_{m \in M,} \sum_{a \in A \cup A^{\prime}} x_{a}^{m} \cdot b_{a}^{m} \\
& \text { s.t. (2),(3),(5),(6),(7),(8),(10). } \\
& \text { (16) }
\end{aligned}
$$

\subsubsection{Establishing the Primal Sub-Problem}

Given a feasible initial binary solution $\left(y_{a}\right)$ to start with, the original problem (16) for the first iteration $(i=1)$ is solved to return traffic volume $\left(x_{a}\right)$ as well as $\omega_{a} ; a \in A^{\prime}$ Lagrange multipliers associated with the inequality (8). Hence the Lagrangian objective function can be written as $^{9}$ :

$$
\begin{gathered}
L\left(x_{a}, y_{a}, \omega_{a}\right)=\sum_{a \in A} x_{a} t_{a}\left(x_{a}\right)+\sum_{a \in A^{\prime}} x_{a} t_{a}\left(x_{a}\right)+ \\
\sum_{m \in M, a \in A \cup A^{\prime}} x_{a}^{m} \cdot b_{a}^{m}+\sum_{a \in A^{\prime}} \omega_{a}\left(x_{a}-U y_{a}\right) \\
\text { (17) }
\end{gathered}
$$

The Lagrange function can be rearranged as follows:

$$
\begin{aligned}
& L\left(x_{a}, y_{a}, \omega_{a}\right)=\sum_{a \in A} x_{a} t_{a}\left(x_{a}\right)+\sum_{a \in A^{\prime}} x_{a}\left(\omega_{a}+t_{a}\left(x_{a}\right)\right)+ \\
& \sum_{m \in M, a \in A \cup A^{\prime}} x_{a}^{m} \cdot b_{a \in A^{\prime}}^{m}-\sum_{a} U y_{a}
\end{aligned}
$$

\footnotetext{
${ }^{9}$ Note that problem (17), is derived based on problem (16) via a feasible binary solution. Eqs $(2,3)$ contain binary variables which stands for the feasibility, hence both are automatically dissolved when a feasible binary solution is arrived at. As such there is no point to bring them into the dual problem.
}

where $U$ is a sufficiently large value hence, if Constraint (8) is found binding (or $\omega_{a}=0$ ), we get $y_{a}=1$, otherwise we have $\omega_{a}>0$ for $y_{a}=0$. Therefore a complementarity constraint always holds $y_{a} \cdot \omega_{a}=0$, or equivalently $x_{a} \cdot \omega_{a}=0$. Accordingly, the last term in Equation (18) vanishes. Given a feasible solution $y_{a}$ we have:

$$
\begin{gathered}
U B_{b}^{i}=\operatorname{Min} L\left(x_{a}, \omega_{a}\right)=\sum_{a \in A} x_{a} t_{a}\left(x_{a}\right)+ \\
\sum_{a \in A^{\prime}} x_{a}\left(\omega_{a}+t_{a}\left(x_{a}\right)\right)+\sum_{m \in M, a \in A \cup A^{\prime}} x_{a}^{m} \cdot b_{a}^{m}
\end{gathered}
$$

s.t. (5),(6),(7),(10)

The above problem has simply become a capacitated MMMC-SO traffic assignment while the only additional component is omega $\left(\omega_{a} \geq 0\right)$.

As can be seen, the omega needs to be added to the travel time of the respective project. This problem is still convex and can be solved by Augmented Lagrangian Method (ALM) (Larsson and Patriksson, 1995; Patriksson, 1994) or Inner Penalty Function (IPF) (Nie et al., 2004). There are some challenges in both methods such as the number of parameters involved.

Alternatively, the authors have recently proposed a method based upon an intuitive interpretation of the omegas (lagrangian values) for the general Capacitated Traffic Assignment Problem (C-TAP). In the objective function of the above problem, the omegas sit next to the delay term. Thus the omegas can be treated as a penalty term to be imposed on the candidate projects (roads), those who have been decided as no-build in order to block them. This interpretation leads to a method dubbed "Inflated Travel Time" which bears none of the aforementioned shortcomings in AFW and IPF methods (Bagloee and Sarvi, 2015b). As a result, the C-TAP is transformed into a normal, incapacitated TAP for which any conventional methods such as Frank-Wolfe are applicable.

The value of the objective function in formulation (19) denoted by $U B_{b}^{i}$ is the total travel time of the MMMC-SO traffic flow, which renders an upper bound at iteration $i$ (subscript $b$ refers to Benders to distinguish this upper bound from the upper bound of the Branch-and-Bound).

So far, the Primal Sub-Problem which returns $\left(x_{a}^{i}, \omega_{a}^{i}\right)$ is solved. We can now proceed to establish the Relaxed Master Problem through which we seek new binary decision values for the next iteration $\left(y^{i+1}\right)$.

\subsubsection{Establishing the Relaxed Master Problem}

Considering the feasible solution that resulted from solving the previous Primal Sub-Problem, let us now rewrite the original as follows. As discussed before, the dual (Lagrangian) format of 
the original problem (OP) is established based upon the solution that results from the primal Subproblem $\left(x_{a}\right): L\left(x^{i}, y, \omega^{i}\right)=f\left(x^{i}, y\right)+\omega^{i} . S\left(x^{i}, y\right)$. Note that the objective function of the OP (equation (16)) is free from any binary decision variables $(y)$, hence $f\left(x^{i}, y\right)=f\left(x^{i}\right)$ becomes the total travel time of the MMMC-SO traffic flow, which is already computed and denoted by $U B_{b}^{i}$. As for the second term $\left(\omega^{i} . S\left(x^{i}, y\right)\right)$, given the already specified values of $x_{a}^{i}$, the only constraint left in the OP is constraint (8). Using $\omega_{a}^{i}$ as the dual variable for constraint (8), we can then bring it up to the objective function using the penalty term: $\omega_{a}^{i} \cdot\left(x_{a}^{i}-U \cdot y_{a}\right)$. Remember that we have already shown the following complementarity relationship: $\omega_{a}^{i} \cdot x_{a}^{i}=0$. Hence, by summing up over $a \in A^{\prime}$, the second term is obtained as $-U \cdot \sum_{a \in A^{\prime}} \omega_{a}^{i} \cdot y_{a}$. Consequently, the dual lower bound to the OP (Benders cuts) can be finalized as: $V \geq U B_{b}^{i}-U \cdot \sum_{a \in A^{\prime}} \omega_{a}^{i} \cdot y_{a}$ where $V$ is the value of the objective function of the relaxed master problem. The above cut, along with the other cuts, stacked up in the previous iterations are combined and are included in the relaxed master problem as follows:

$$
\begin{aligned}
& L B_{b}^{i}=\min _{y_{a}} V \\
& \text { (20) } \\
& \text { S.t. } \quad V \geq U B_{b}^{k}-U . \sum_{a \in A^{\prime}} \omega_{a}^{k} \cdot y_{a}, k=1 . . i, \\
& \sum y_{a}-\sum y_{a} \leq\left|Y 1^{k}\right|-1, \\
& \text { (21) } a \in Y 1^{k} \quad \sum_{k \in Y 0^{k}} \quad \text { where } \\
& Y 1^{k}=\left\{a \mid y_{a}^{k}=1\right\} ; Y 0^{k}=\left\{a \mid y_{a}^{k}=0\right\}, k=1 . . i \text {, } \\
& \text { (22) } \\
& \sum_{a \in A^{\prime}} c_{a} \cdot y_{a} \leq B
\end{aligned}
$$

where constraints (22) and (23) ensure rendering a new and feasible binary variable $\left(y_{a}^{i}\right)$ solution at each iteration. So far, both primal and relaxed problems are established and we now proceed to establish the Benders decomposition algorithm:

Step 0 (Initialization)- set iteration counter $i:=1$; $U B_{b}^{i}:=+\infty$; set initial solution for binary variables $\left(y_{a}^{i}=0\right)$; set convergence gap $\varepsilon$

Step 1- Given $y_{a}^{i}$ solve the primal problem (19) to find $U B_{b}^{i}, x_{a}^{i}, \omega_{a}^{i}$. Set $U B_{b}^{*}=\min \left(U B_{b}^{i}, U B_{b}^{*}\right){ }^{10}$

\footnotetext{
${ }^{10}$ The algorithm starts with an initial solution, based on which a traffic assignment is calculated and values of $x_{a}$ are found if a project is decided not to be constructed for which we then have $x_{a}=0$
}

Step 2- Give $x_{a}^{i}, \omega_{a}^{i}$ solve the Relaxed Master Problem (20) to (23) to find $L B_{b}^{i}, y_{a}^{i}$

Step 3 (termination) if $\left(U B_{b}^{*}-L B_{b}^{i}\right) / L B_{b}^{i} \leq \varepsilon$ then the convergence is achieved so it returns $U B_{b}^{*}$ as the final solution. Otherwise $i:=i+1$; go to Step 1

In order to better understand how Benders decomposition works, a simple example is undertaken as presented in Appendix A. The merit index is an educated guess so as to quickly arrive at the optimum solution. It is based on an intuitive view on the optimum solution; that is, if a road is really necessary, upon completion of construction, it must become highly congested. We have solved the example provided in Appendix A with and without a merit index. The results are presented in Appendix B which shows that the search for the optimal solution is highly decreased.

Before proceeding to the next section dedicated to the numerical evaluations, there are some remarks which are discussed in Appendix B. Although an attempt was made to preserve the integrity of the manuscript, the interested reader may want to read the appendix before proceeding to the numerical evaluations.

\section{NUMERICAL EVALUATIONS}

In this section, we first examine no-frills (unimodal and single class) versions of the algorithm over Gao's 12-nodes network (Gao et al., 2005) and the Sioux Falls benchmark network (Farvaresh and Sepehri, 2013; Leblanc, 1975) to cast the proposed methodology in the context of its peers in the literature. We then apply the algorithm in its full capacity (multiclass and multimodal) to the large scale network of the city of Winnipeg, Canada. Exhaustive enumerations have already been carried out to find optimal solutions for all the case studies that have been undertaken over various budget levels. The enumeration entails all combinations of network scenarios with accounts for solving $1,048,576\left(=2^{\wedge} 20\right)$ traffic assignment problems. The quality of the solutions resulting from the proposed algorithm will be compared against the optimal solutions.

Parameters setup of the algorithm is as follows: (i) the relative gap introduced in Benders algorithm, Step 3 is assumed: $\varepsilon=2 \%$ (ii) the value of $\alpha$ introduced in Eq (25) is initiated with zero to seek the tightest possible lower bound. Since $\alpha=0$ 
might compromise the quality of the solutions; should the optimal solution not found ${ }^{11}$ at $\alpha=0$, more attempts with positive values of the alpha will be tested.
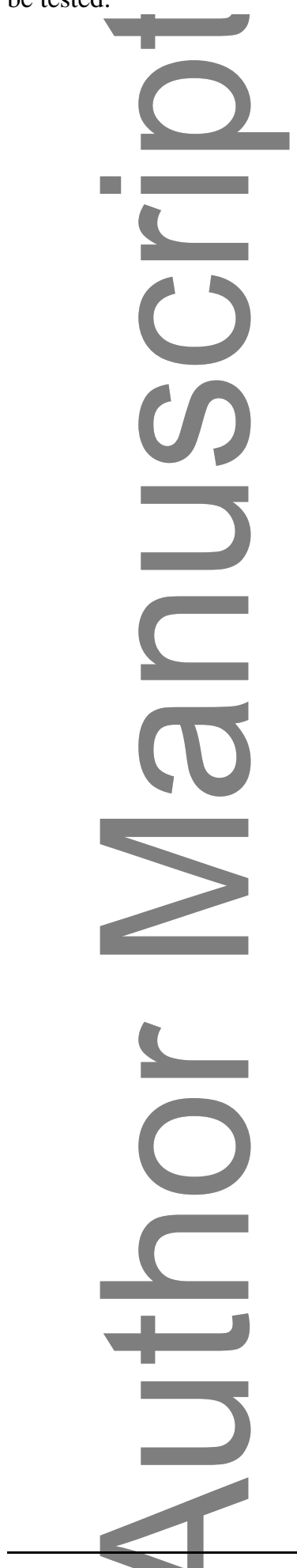

${ }^{11}$ As noted early, for the undertaken case studies the optimal solutions have already been identified via the enumeration. We first run the algorithm with alpha equals to zero. The subsequent result shows that the optimal solutions are likely to be found in early iterations. Hence -in real practice, where the optimal solutions are not known and we are facing with an NP-hard problem - one can still run with alpha-zero and expecting to find a good solution (if not the optimal) at a bearable computational time. 


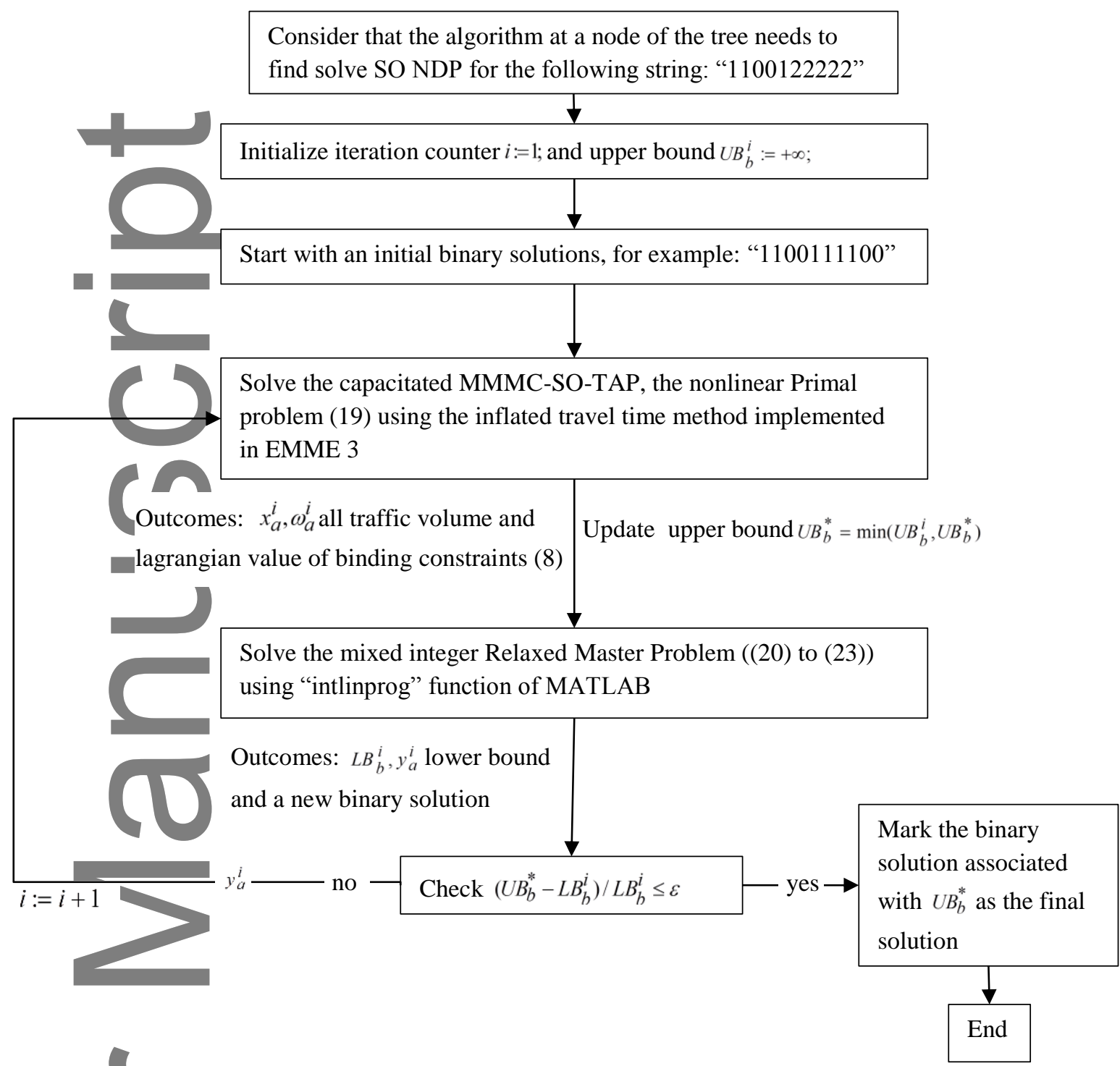

Figure 2 Benders algorithm: the flowchart graphically represents the steps

As discussed before (in Remark 4), the first lower bound is calculated for the root node of the B\&B's tree (representing string " $22 . .22$ "). In most of the numerical tests, the optimal solutions were found at the root node. As a result, in the comparative analysis for the Gao network and the Sioux-Falls benchmark, the focus is set on the number of iterations made at the root node to arrive at the optimal solution. A comparison will be made against some of the state-of-the-art exact methods in past studies. In light of the fact that the CPU time is heavily subject to the computational technology used at the time, as well as the coding architect, the number of iterations can be regarded as a fair yardstick in comparative analysis. Furthermore, each iteration of exact methods is usually involved in alternatively solving two subproblems (UE problem and MILP) which is analogous to the proposed method in this study. Nevertheless, the algorithm quickly terminated for the Gao's 12-nodes network and the Sioux-Falls network in a matter of a few seconds and a few minutes respectively. Furthermore, the CPU time for solving the large scale Winnipeg network will be discussed further in detail.

For all the case studies, in addition to optimal solutions and the corresponding objective function value (i.e. the total travel time is dubbed as the incumbent value), the size of the solution spaces (i.e. the number of feasible solutions) are also presented. The size of the solution spaces gives us

This is the author manuscript accepted for publication and has undergone full peer review but has not been through the copyediting, typesetting, pagination and proofreading process, which may lead to differences between this version and the Version of Record. Please cite this article as doi: $\underline{10.1111 / \text { mice. } 12224 .}$

This article is protected by copyright. All rights reserved. 
an indication of how rough and unlikely the path toward the global optimum solution may be.

As for the computational technology, we employ a desktop computer with $\operatorname{Intel(R)} \operatorname{Xeon}(\mathrm{R}) 3.70$ $\mathrm{GHz}$ and 64.0 GB RAM. The algorithm is coded with Visual Basic linked to MS-Excel and MSAccess as an interface and save/retrieve database. The computer code is also synchronized with EMME 3 to solve the multimodal and multiclass traffic assignment problems. The code also calls on MATLAB 14a to solve the MILP problems using the newly released module "intlinprog" (MathWorks, 2014). All delay functions associated with the links conform to the BPR type.

For the Sioux-Falls and Winnipeg case studies, the candidate projects are two-way roads that are found in the real world. Should a candidate project receive approval for construction, two directional links need to be added to the network. Instead of representing each directional link as a separate decision variable (which leads to an increase in the number of binary variables and constraints), we propose the concept of a "directional switch link" to represent the two-way roads. Figure 3 illustrates a two-way road between $\mathrm{A}$ and $\mathrm{B}$ at the top. The same two-way road is disconnected into two pieces (without change to any characteristics) and is then reconnected with a one-way switch link (the dashed line) at the bottom (the two-way links are shown as bended outward to better illustrate the switch link). In this way, the switch link can represent the twoway links (painted by red and green colours).

What then needs to be done is to code the disconnected two-way roads in the base network scenario. All components of the disconnected links, including the switch links, have a zero value for travel time, except the two segments that correspond with the two directions. These two segments inherit all of the specifications of the original direction such as length, travel time and the number of lanes, etc as denoted by $t_{\mathrm{AB}}$ and $\mathrm{t}_{\mathrm{BA}}$ in Figure 3. Should a decision be made to construct a (two-way road) project (i.e. $y=1$ ), the corresponding one-way switch link is then added to the network to reconnect the two-way road. The concept of a switch link can also be used to represent more complicated projects such as spaghetti interchanges.

6.1. Example 1: Gao's network

Figure 4 illustrates the example network developed by Gao et al. (2005) with one OD pair $(1,12)$ and the travel demand of $q_{1,12}=20$. The delay function is $t_{a}=\bar{t}_{a}+.008 \cdot x_{a}{ }^{4}$, and it can be rearranged as per the BPR format: $t_{a}=\bar{t}_{a}\left(1+.15\left(x_{a} / w_{a}\right)^{4}\right)$ where the links capacities are $w_{a}=\sqrt[4]{.15 \bar{t}_{a} / .008}$. There are six candidate (oneway) roads with a total cost of 70. Gao et al. (2005) developed and applied the Generalized Benders
Decomposition (GBD) to various budget levels and the results are summarized in Table 1.

Table 1 shows total number of iterations and the iteration at which the optimal solution was found in Gao's GBD method as well as the proposed B\&B$B$ algorithm. As can be seen across all budget levels, the B\&B-B demonstrates significantly superior performance over Gao's GBD. Furthermore, the B\&B-B on two avenues of the objective functions (with/without budget consumption term) showed close results. Since the proposed algorithm (as proven before) guarantees the optimum solutions, the merit index is devised to accelerate the algorithm. For instance as Table 1 suggests, in terms of the number of iterations, Gao's GBD (which lacks any merit index) lags behind in all budget levels.

\subsection{Example 2, Sioux-Falls network}

The Sioux-Falls dataset was first introduced by (Leblanc, 1975), and a slightly modified version was recently used by Farvaresh and Sepehri (2013) employing a Branch-and-Bound and Outer Approximation (B\&B-OA) method. In this section, we compare the application results of our study with the results reported by Farvaresh and Sepehri (2013) on the same Sioux-Falls network. There are five two-way candidate roads with a total cost of 4325. In a similar fashion, Table 2 presents the comparative results. As is evident from Table 2, in this case the proposed B\&B-B surpasses the B\&BOA.

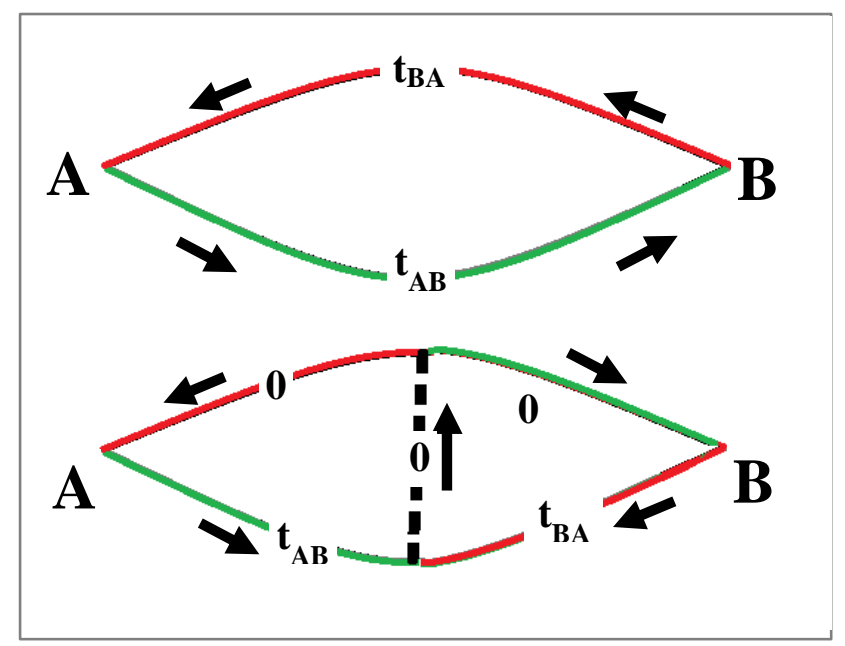

Figure 3, A one-way switch link (dashed line) representing a two-way road (red and green lines)

It was in only one out of three budget levels (pertaining to the "without the budget consumption") in which B\&B_B was found slightly lagging behind B\&B-OA. Whereas in the presence of the budget consumption term (which is the preferable method), our proposed algorithm (B\&B_B) was by far leading B\&B-OA. Nevertheless, the spirit of the literature suggests that it is unlikely to arrive at an absolute and 
superior algorithm. The important point is that for the first time, the proposed algorithm is purposely tailored to large sized networks. So when it is applied to small and artificial benchmark networks, the proposed algorithm has also shown a relative superiority.

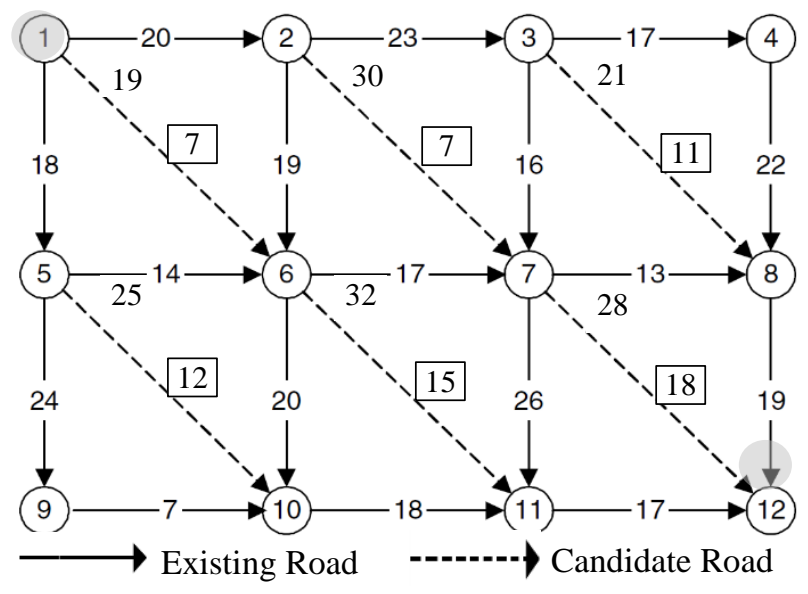

$19 \bar{t}_{a}$ Free flow travel time (1) Node number

$7 c_{a}$ Candidate's construction cost

Figure 4 Gao's test Network

\subsection{Example 3: Winnipeg large-scale network}

Real-size transportation data for the city of Winnipeg, Canada is widely used in the literature (Bagloee and Asadi, 2015; Bagloee and Tavana, 2012; Bar-Gera, 2015; Ryu et al., 2015) and is undertaken for numerical tests considering multimodal and multiclass traffic assignment. This dataset has also been provided in EMME 3 (INRO, 2009). The road network is comprised of 154 zones, 943 nodes and 3075 directional links. The transit system consists of 2 transit vehicle types, 133 transit lines and 4345 transit line segments.As with the multiclass aspect of traffic flow, in addition to the inclusion of different types of vehicles (truck, car, etc), the bias term can be applied to many other real life applications such as traffic restriction, High Occupancy Lanes (HOV) and toll gates, etc. (INRO, 2009). For instance, in the case of $b_{a}^{m}=\infty$ the respective user class $m$ is prevented from entering the district denoted by link $a$. Accordingly, the same dataset that was used for the single-class Winnipeg case study is also used (but split between) the Central Business District (CBD) and non-CBD which resulted in two travel demand matrices. The CBD matrix which accounts for 19,742 vehicle trips can use all roads including roads in the CBD. The non-CBD commuters $(36,476$ vehicle trips) are prohibited in the CBD. The location of the CBD is shown in Figure 5. The transit demand contains 18,211 passenger trips. In the existing scenario, the average travel time of the non-CBD commuters is 13.62 minutes while it is 17.10 minutes for the CBD bound commuters (CBD bound trips are the ones that have at least either their origin or their destination falling inside the CBD). As such, the average in-vehicle time experienced by passengers is 22 minutes.

Table 1 Example 1; Gao's Network: GBD (Gao et al., 2005) versus proposed B\&B-B

\begin{tabular}{|c|c|c|c|c|c|c|c|c|c|}
\hline \multirow[b]{3}{*}{ Budget $^{1}$} & \multirow[b]{3}{*}{$\begin{array}{ll}\text { Optimal }^{2} & \text { of } \\
\text { solution } & \text { feasible } \\
& \text { solutions }\end{array}$} & \multirow[b]{3}{*}{$\begin{array}{l}\text { Incumbent } \\
\text { Value }\end{array}$} & \multirow{3}{*}{\begin{tabular}{l}
\multicolumn{1}{c}{ GBD method ${ }^{3}$ : } \\
\\
Total iteration \\
(Optimum solution \\
was found at \\
iteration)
\end{tabular}} & \multicolumn{6}{|c|}{ Proposed; B\&B_B method ${ }^{5}$} \\
\hline & & & & \multicolumn{3}{|c|}{ With budget consumption term: } & \multicolumn{3}{|c|}{ Without budget consumption } \\
\hline & & & & $\begin{array}{l}\text { no of UE } \\
\text { solved }^{6}\end{array}$ & $\begin{array}{l}\text { no of } \\
\text { Benders } \\
\text { (lower } \\
\text { bound) } \\
\text { solved }^{6}\end{array}$ & $\begin{array}{l}\text { Benders } \\
\text { iteration at } \\
\text { which } \\
\text { optimum } \\
\text { solution } \\
\text { was found }\end{array}$ & $\begin{array}{l}\text { no of UE } \\
\text { solved }^{6}\end{array}$ & $\begin{array}{l}\text { no of } \\
\text { Benders } \\
\text { (lower } \\
\text { bound) } \\
\text { solved }^{6}\end{array}$ & $\begin{array}{l}\begin{array}{l}\text { Total } \\
\text { iteration }\end{array} \\
\text { (Optimum }^{6} \\
\text { solution } \\
\text { was found } \\
\text { at } \\
\text { iteration) } \\
\end{array}$ \\
\hline 10 & 100000 & 4076 & $3(2)$ & 3 & 2 & $0^{4}$ & 3 & 2 & $0^{4}$ \\
\hline 20 & 101 & 3952 & $6(4)$ & 3 & 5 & $0^{4}$ & 3 & 5 & $0^{4}$ \\
\hline 30 & 100001 & 2668 & $7(6)$ & 4 & 3 & 2 & 4 & 3 & 2 \\
\hline 40 & 100101 & 2524 & $9(4)$ & 4 & 5 & 2 & 4 & 5 & 2 \\
\hline 50 & 101101 & 2404 & $10(4)$ & 4 & 6 & 5 & 4 & 6 & 3 \\
\hline 60 & 1011 & 2281 & $8(5)$ & 4 & 5 & 2 & 4 & 6 & 5 \\
\hline 70 & 111111 & 2256 & $5(5)$ & 3 & 1 & $0^{4}$ & 3 & 1 & $0^{4}$ \\
\hline
\end{tabular}


them. Instead we report the number of iterations broken down in number of times that Benders were solved plus number of times that a traffic assignment was solved. The former is comparable with the number of iterations reported by Gao, since in both, two primal and relaxed problems are alternatively solved. So "total iteration" in the B\&B_B refers to number of times Benders is solved.

Table 2, Example 2; Sioux-Falls: B\&B-OA (Farvaresh and Sepehri, 2013) versus proposed B\&B-B

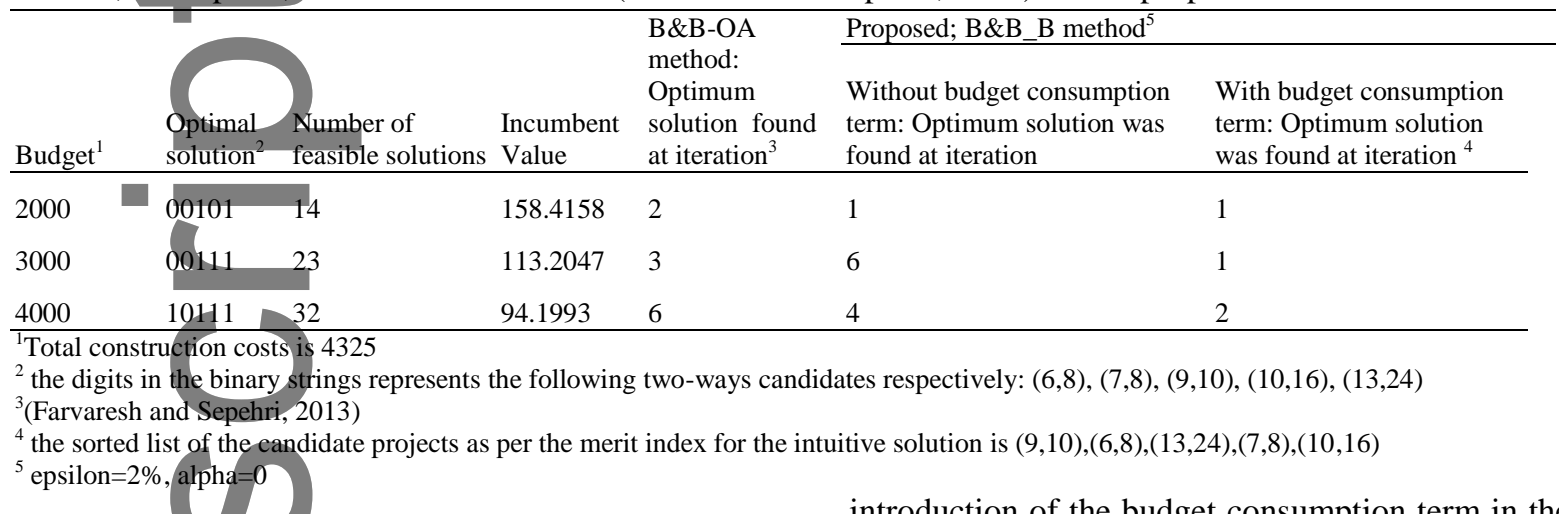

We will consider twenty (20) two-way road projects with speeds of $50 \mathrm{~km} / \mathrm{h}$ (or an equivalent free flow time of $60 *$ length $/ 50$ minutes) and a capacity of 1700 vphpd ${ }^{12}$. Table 3 presents the candidate projects sorted and based on their merit index in descending order. Figure 5 shows the location of the candidate projects and the extent of the undertaken case study on which MMMC UE traffic volumes are depicted (all projects, irrespective of any budget constraints, are included). These projects are wisely set forth, to complement the ring roads around the CBD and over the river passing through the city. The length of roads will be considered with corresponding construction costs which amount to the total monetary cost of $\mathrm{C}=23.31$. With respect to the total cost (C), we take five levels of budget (B) into analysis as follows: $\mathrm{B} / \mathrm{C}=20 \%, 40 \%, 60 \%, 80 \%$ and $100 \%$. The MMMC UE traffic assignment is solved with a relative gap of $.001 \%$ where each traffic assignment lasts approximately 3 seconds.

In the previous two examples, the budget consumption term was a contributing factor in reaching a global solution faster13. Accordingly, in the Winnipeg case, we carried out the analysis only on scenarios in the presence of the budget consumption terms. Consequently, the numerical results of the $\mathrm{B} \& \mathrm{~B}-\mathrm{B}$ pertaining to the objective functions equipped with the budget consumption terms are reported in Table 4 . In Table 4, global optimal solutions are first introduced over various budget levels. As we can see, the global optimal solutions have used up almost all of the budgets, such that the budget consumption varies from $89 \%$ to $99 \%$. As a result, it is an endorsement of the

\footnotetext{
${ }^{12}$ vehicle per hour per direction

${ }^{13}$ The budget consumption helps us to arrive at an educated guess for the optimum solution. Since it is merely a guess, there is no proof that it will always work favourably. Nevertheless in Table 1 , in only one out of seven cases and zero out of three cases in Table 2, the budget consumption did not hit the target. Therefore, the likelihood of failure of the budget consumption is very low (in these cases, only $10 \%$ or 1 out of $7+3$ ).
}

introduction of the budget consumption term in the objective function. In other words, encouraging the algorithm to use up the full capacity of the available budget results in and contributes to global solutions being arrived at quicker. A closer look at the binary string of the global solutions over the incremental budget levels suggests that the strings incline so that they are filled from left to the right. This observation underscores the validity of the merit index in an attempt to enhance the likelihood of finding the global solution. According to Table 4 , for each budget level we start with $\alpha=0$. In two out of the five budget levels, the global optimal solutions were found. In the remainder, good solutions ranked 4th, 6th and 3rd were achieved. Consideration of a meagre value for the alpha (i.e. $\alpha=0.001$ ) secured global solutions for budget levels $40 \%$ and $100 \%$. It was only for budget level $80 \%$ where $\alpha=0.001$ enhanced the quality of the solution from 6 th to 4 th. In addition, a slight push on the alpha from $\alpha=0.001$ to $\alpha=0.005$ resulted in the global optimal solution ${ }^{14}$.

\footnotetext{
${ }^{14}$ Although compared to Leblanc's lower bound, the systemoptimal (SO) network design problem proposed in our study is generally tighter, it may come at higher computation costs. Furthermore, no matter how tight the SO lower bound might be, there is no guarantee that one will find the SO's lower bound higher than the best upper bound value (incumbent value). This would be even more likely for Leblanc's lower bound. If such things happen, the proposed methodology becomes a simple enumeration. In order to lower the likelihood of such cases, we introduce the concept of alpha (equation (25)). Alpha varies between 0 and 1: if it gets close to zero, the $\mathrm{SO}$ is approximated by the UE. In the numerical results, we show that even a trivial value for alpha does a very good job in the sense that the frequency of finding the SO lower bound higher than the incumbent value increases, while finding the global optimum solution is not compromised. Furthermore, we replaced the SO lower bound module by a simple SO traffic assignment (Leblanc lower bound) and run it for the Winnipeg network subject to the budget level of $\mathrm{B} / \mathrm{C}=20 \%$. Unfortunately at none of the branches was the lower bound found to be higher than the incumbent value and the computation time elapsed 7.02 hours: seven times higher than our proposed algorithm (see Table 4); we repeated the same run for $\mathrm{B} / \mathrm{C}=40 \%$, after 12 hours and unfortunately no good lower bound was found so we terminated the process.
} 
Table 4 also indicates the number of times and the depth at which fathoming occurred, both of which are a key efficacy barometer. The $11^{\text {th }}$ column ("Total budget consumption before fathoming") shows the average depth of the fathoming point in a percentage of the budget. The less depth is more desirable since it suggests that the fathoming has occurred at the beginning of the tree structure; hence a big portion of the solution space has been discarded.

The computational (CPU) time has also been reported, such that in the worst case $(B / C=40 \%$, alpha=.001), the computation lasts almost 6.6 hours. The last column in the table represents the percentage of the total CPU time at which the best solutions are found, and this can vary from very early $(2 \%)$ to almost half way through $(62 \%)$. In six out of ten budget levels, optimal solutions were found in the first half of the CPU time. These percentages show that there is a high probability of reaching the best solution in early iterations. Furthermore, the number of attempts to solve Benders problem (MMMC-SO-TAP with Lagrangian value of the capacity constraints as well as MILP) and MMMC-UE-TAP are also reported in the table. Such breakdown detail gives us a better understanding of the sheer size of the computational burden over different sized networks. For instance, (Xie and Xie, 2014) have recently reported on CPU times for solving various network sizes which varies from a few seconds to a few minutes for super large scale local networks (with +1770 zones). In dealing with super largesize networks, instead of a couple of hours (which was the case for the Winnipeg network), one may need to wait a couple of days to successfully terminate the algorithm. It is worth noting that for strategic planning decisions such as network design which involves hefty investment, one can afford CPU time in the scale of hours or even days.

In a nutshell, compared to the do-nothing scenario, the improvements corresponding to the scenario with maximum number of projects $(\mathrm{B} / \mathrm{C}=100 \%$ : 18 out of 20 projects are added) were achieved as follows: the total travel time (all modes and vehicle classes combined) improved $3.1 \%$ (objective functions of 839,462 versus 866,499 ). Furthermore, the travel time of two vehicle classes of CBD bound and non-CBD were found 16.53 and 13.13 minutes which are equivalent of almost 3.3 $\%$ compared to those of the do-nothing scenario. While the projects were initially aimed to improve the private modes, the transit mode also received $0.6 \%$ improvement in transit time.

\section{Conclusions}

In this study, we have approached the discrete network design problem (DNDP) via an exact algorithm tailored to the need, scale and nature of the problems dealt with in the industry. Although the DNDP has been extensively studied in academia, practitioners are yet to find anything appealing in it. There is a two-fold reason for this: (i) the size of the realistic problems and the combinatorial nature of the DNDP are a significant prohibitive factor; and (ii) in spite of the existence of efficient heuristic (but not exact) methods (mainly due to their non-deterministic nature), neither practitioners nor the commercial software likes them.

The ongoing growth of infrastructure in Asia (Estache et al., 2013) on the one hand and the enhancement in computational power as well stateof-the-art optimization techniques on the other hand, have raised an interest in filling the gap between academia and industry via exact (not heuristic) methods. In spite of the fact that the DNDP is an NP-hard problem, the size of the problems being dealt with in the industry is intractable (say a dozen candidate projects) ${ }^{15}$. All together, these make addressing the DNDP on realistic scales a worthwhile endeavour. To this end, we propose a $B \& B-B$, a method based on Branch and Bound (B\&B), hybridized with Benders decomposition to streamline achieving a global optimum solution. Using a set of greedy rules, the tree of the B\&B was built upon the sorted projects on a merit basis, aiming at reaching the global optimum solution. In order to hedge against the Braess Paradox, the total travel time of the System Optimal (SO) flow was computed as a lower bound at each new node on the tree (Leblanc, 1975). To further reduce the computation time, a Benders method is devised to obtain a tight lower bound. The algorithm proposed was evaluated numerically using a real data set from the city of Winnipeg, with 20 candidate projects subject to various budget levels. In the context of the past studies, we have also provided comparative analysis. Accordingly, the proposed B\&B-B has shown superior performance.

The algorithm proposed could be further improved by considering asymmetric delay functions to enhance the degree of realism of the traffic flow. In the same spirit, consideration of changes in travel demand in response to changes in the network side (known as demand elasticity) is a worthwhile thread for research in the context of NDP. Furthermore, the conventional wisdom is to represent the congestion using the delay functions

\footnotetext{
${ }^{15} \mathrm{~A}$ recent literature review shows that number of projects even in the heuristic-based approaches revolves around 20+ (Farahani, R.Z., Miandoabchi, E., Szeto, W., Rashidi, H., 2013. A review of urban transportation network design problems. European Journal of Operational Research 229, 281-302., see Table 7 and listed papers therein). Furthermore, the first author was lately involved in a mega road extension plan for the city of Dubai, United Arab Emirates, known as "R956 project" in which 17 road construction projects were evaluated, subject to different budget scenarios.
} 
and Beckmann formulation. There are two areas that can improve the congestion representations: (1) considering the limited capacity of the roads; and (ii) considering the dynamic changes of the congestion. Dynamic Traffic Assignment (DTA) is an evolving subject that takes the above concerns into consideration. As a result, integration of the DNDP and DTA can also be of interest for future studies.

The NDP is based on a concrete premise that the candidate projects are given. Loosening such a premise and arriving at a more sophisticated way to find the candidate projects in conjunction with the NDP is worth further research.

Furthermore, it is sometimes imperative to arrive not only at the best (global) optimal solution $\left(1^{\text {st }}\right)$, but at other good solutions belong to the top of the list (say $2^{\text {nd }}, 3^{\text {rd }}, 4^{\text {th }}$ etc). Given many different stakeholders and vested interest in the transport infrastructure, providing decision makers with a variety of top-performing solutions is incredibly appealing in the industry. In some cases, the difference between the $1^{\text {st- }}$ and $2^{\text {nd- }}$ best solutions in terms of the objective function is only mathematically marginal, but the $2^{\text {nd }}$ best solution has some other advantages which cannot be quantified in the objective function. Similar to the terminology used in the optimization literature $(\mathrm{k}$ shortest paths), we call this new problem K-NDP, where the intention is to find the first " $k$ " best solutions.

Extending the discussion to selecting an optimum configuration of one-way and two-way roads is also a worthwhile line of research (Drezner and Wesolowsky, 1997, 2003). Concern arose out of the vested interests involved in the road network and this may bring about a variety of objective functions in the DNDP including environmental costs (Szeto et al., 2014) and emission reduction (Ferguson et al., 2012). As such, the problem becomes a multi-objective DNDP (Xie, 2014). Changes to the network in the short and long run may affect the way commuters choose their mode of transport, their destination and departure time (if any). Hence, the DNDP subject to a combined traffic model considering these changes deserves more investigations (Boyce and Janson, 1980; Boyce and Soberanes, 1979). Similarly investigation of land use changes as the result of changes in the network infrastructure can also be noted (Szeto et al., 2010; Szeto et al., 2013). Although the consensus in the literature is to ignore the uncertainty of the travel demand, one may want to explore such uncertainties involved in the DNDP (Ukkusuri et al., 2007).

The NDP can also be extended to traffic control and management such as ramp metering and priority measures (Bagloee et al., 2016; Mesbah et al., 2011a; Mesbah et al., 2011b; Sarvi and Kuwahara, 2008; Sarvi et al., 2004).

\section{ACKNOWLEDGEMENT}

The authors are very grateful to Prof Adeli and eight anonymous reviewers for their meticulous, insightful and highly constructive comments which greatly contributed to the content and presentation of the article. Presentation of the article was further improved based on the comments received from Prof Jeff Sellar from Monash University, Australia.

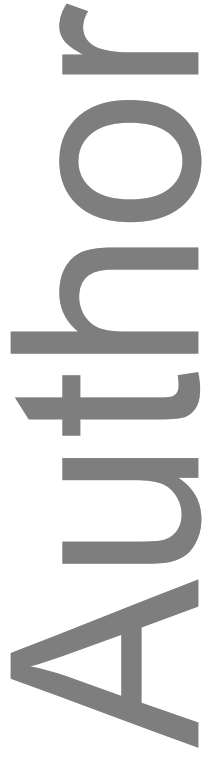




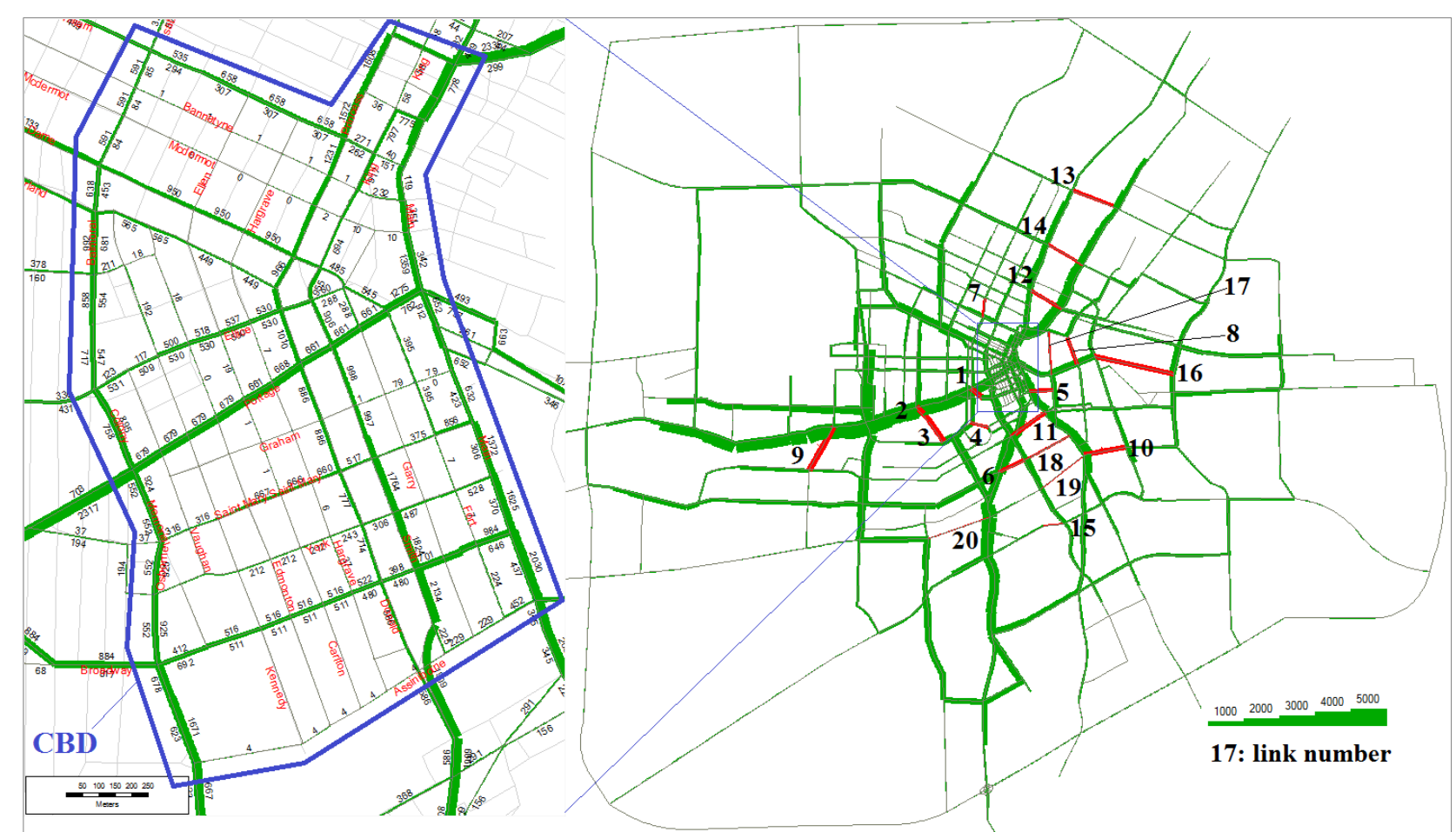

Figure 5 Winnipeg Network, The 20 road projects, Central Business District (CBD) and multiclass multimodal traffic volume plot.

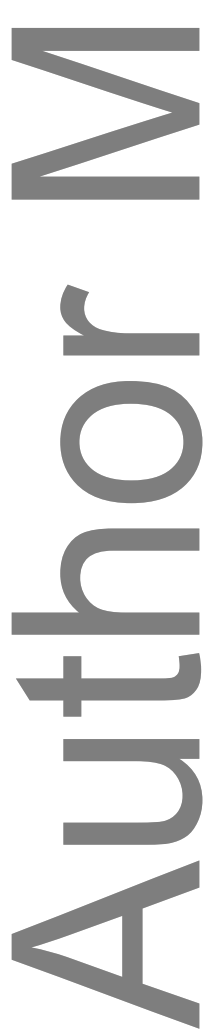

This is the author manuscript accepted for publication and has undergone full peer review but has not been through the copyediting, typesetting, pagination and proofreading process, which may lead to differences between this version and the Version of Record. Please cite this article as doi: 10.1111/mice.12224.

This article is protected by copyright. All rights reserved. 
Table 3 Winnipeg Case Study, two-way (candidate) road projects sorted based on the merit index

\begin{tabular}{|c|c|c|c|c|c|c|c|c|c|c|c|c|c|c|c|c|c|c|c|c|}
\hline Id & 1 & 2 & 3 & 4 & 5 & 6 & 7 & 8 & 9 & 10 & 11 & 12 & 13 & 14 & 15 & 16 & 17 & 18 & 19 & 20 \\
\hline I-node & 887 & 595 & 513 & 420 & 1035 & 437 & 774 & 1057 & 551 & 325 & 304 & 168 & 177 & 829 & 335 & 288 & 299 & 424 & 330 & 441 \\
\hline J-node & 8 & 602 & 505 & 592 & 301 & 424 & 739 & 297 & 610 & 330 & 423 & 784 & 853 & 173 & 449 & 294 & 1058 & 327 & 428 & 494 \\
\hline $\operatorname{Cost}^{1}$ & 04 & & 0.79 & 0.58 & 0.75 & 0.86 & 0.6 & 0.88 & 1.51 & 1.3 & 1.29 & 1.09 & 1.52 & 1.24 & 0.64 & 2.5 & 1.35 & 1.61 & 1.73 & 2.04 \\
\hline $\begin{array}{l}\text { Traffic } \\
\text { volume }^{2}\end{array}$ & & 554 & 1554 & 822 & 1059 & 1138 & 702 & 1025 & 1688 & 1447 & 1235 & 941 & 950 & 753 & 377 & 1338 & 413 & 469 & 164 & 165 \\
\hline $\begin{array}{l}\text { Merit } \\
\text { Index }^{3}\end{array}$ & 3713 & 2634 & 1967 & 1417 & 1411 & 1323 & 1170 & 1164 & 1118 & 1113 & 957 & 863 & 625 & 607 & 589 & 535 & 306 & 291 & 95 & 81 \\
\hline
\end{tabular}

Table 4 Winnipeg Case Study: results of the proposed B\&B-B

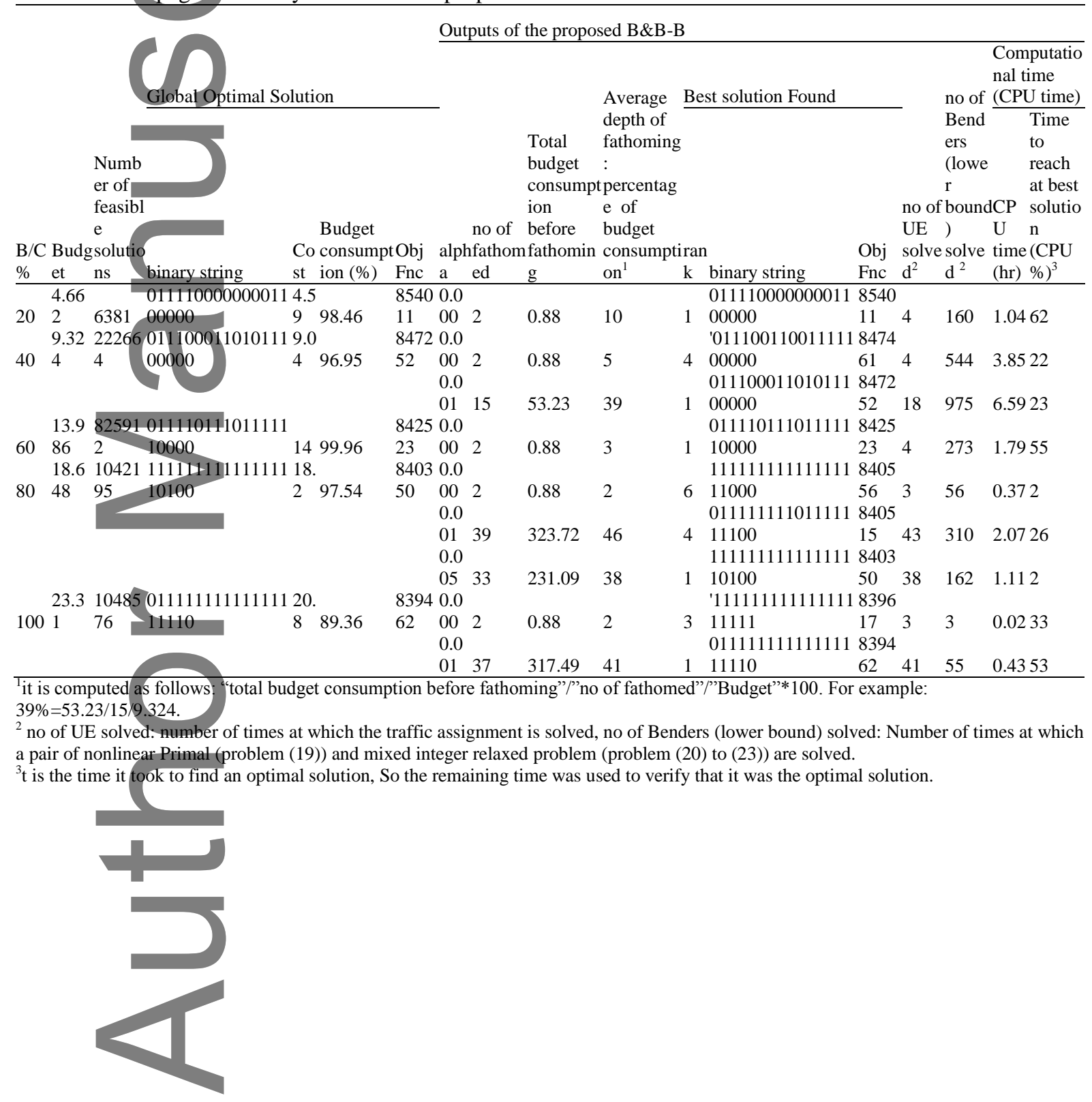

This is the author manuscript accepted for publication and has undergone full peer review but has not been through the copyediting, typesetting, pagination and proofreading process, which may lead to differences between this version and the Version of Record. Please cite this article as doi: $\underline{10.1111 / \text { mice. } 12224 \text {. }}$

This article is protected by copyright. All rights reserved. 


\section{REFERENCE}

Aashtiani, H.Z., 1979. The multi-modal traffic assignment problem. $\mathrm{PhD}$ dissertation, Massachusetts Institute of Technology.

Bagajewicz, M., Manousiouthakis, V., 1991. On the generalized Benders decomposition. Computers $\&$ chemical engineering 15, 691-700.

Bagloee, S.A., Asadi, M., 2015. Prioritizing road extension projects with interdependent benefits under time constraint. Transportation Research Part A: Policy and Practice 75, 196-216.

Bagloee, S.A., Sarvi, M., 2015a. Heuristic Approach to Capacitated Traffic Assignment Problem for Large-Scale Transport Networks. Transportation Research Record: Journal of the Transportation Research Board, 1-11.

Bagloee, S.A., Sarvi, M., 2015b. A Heuristic Approach to Capacitated Traffic Assignment Problem Tailored to Large Scale Networks, Transportation Research Board. Transportation Network Modeling Committee (ADB30); 15-4587, Washington D.C., United States.

Bagloee, S.A., Sarvi, M., Wallace, M., 2016. Bicycle lane priority: Promoting bicycle as a green mode even in congested urban area. Transportation Research Part A: Policy and Practice 87, 102-121.

Bagloee, S.A., Tavana, M., 2012. An efficient hybrid heuristic method for prioritising large transportation projects with interdependent activities. International Journal of Logistics Systems and Management 11, 114-142.

Bagloee, S.A., Tavana, M., Ceder, A., Bozic, C., Asadi, M., 2013. A hybrid meta-heuristic algorithm for solving real-life transportation network design problems. International Journal of Logistics Systems and Management 16, 41-66.

Balakrishnan, A., Magnanti, T.L., Mirchandani, P., 1997. Network design. Annotated bibliographies in combinatorial optimization, 311-334.

Balas, E., Jeroslow, R., 1972. Canonical cuts on the unit hypercube. SIAM Journal on Applied Mathematics 23, 61-69.

Bar-Gera, H., 2015. Transportation Network Test Problems, http://www.bgu.ac.il/ bargera/tntp/.

Bar-Gera, H., Boyce, D., 1999. Route flow entropy maximization in origin-based traffic assignment, 14th International Symposium on Transportation and Traffic Theory. Elsevier Science, Oxford, U.K., Jerusalem, Israel, pp. 397-415.

Beckmann, M., McGuire, C., Winsten, C.B., 1956. Studies in the Eeonomics of Transportation, Yale University Press, New Haven, CT.
Ben-Ayed, O., Blair, C.E., 1990. Computational Difficulties of Bilevel Linear Programming. Operations Research 38, 556-560.

Benders, J.F., 1962. Partitioning procedures for solving mixed-variables programming problems. Numerische mathematik 4, 238-252.

Boyce, D., 2014. Network equilibrium models for urban transport, in: Fischer, M.M., Nijkamp, P. (Eds.), Handbook of Regional Science. Springer Berlin Heidelberg, pp. 759-786.

Boyce, D., Farhi, A., Weischedel, R., 1973. Optimal network problem: a branch-and-bound algorithm. Environment and Planning 5, 519-533.

Boyce, D., Janson, B., 1980. A discrete transportation network design problem with combined trip distribution and assignment. Transportation Research Part B: Methodological 14, 147-154.

Boyce, D., Xiong, Q., 2004. User-optimal and system-optimal route choices for a large road network. Review of network Economics 3.

Boyce, D.E., Soberanes, J.L., 1979. Solutions to the optimal network design problem with shipments related to transportation cost. Transportation Research Part B: Methodological 13, 65-80.

Braess, D., 1968. Über ein Paradoxon aus der Verkehrsplanung. Unternehmensforschung 12, 258268.

Braess, D., Nagurney, A., Wakolbinger, T., 2005. On a paradox of traffic planning. Transportation science 39, 446-450.

Chen, B.Y., Lam, W.H.K., Sumalee, A., Shao, H., 2011. An efficient solution algorithm for solving multi-class reliability-based traffic assignment problem. Mathematical and Computer Modelling 54, 1428-1439.

Chen, M., Alfa, A.S., 1991. A network design algorithm using a stochastic incremental traffic assignment approach. Transportation Science 25, 215-224.

Colson, B., Marcotte, P., Savard, G., 2005. Bilevel programming: A survey. 4OR 3, 87-107.

Colson, B., Marcotte, P., Savard, G., 2007. An overview of bilevel optimization. Annals of operations research 153, 235-256.

Dafermos, S.C., 1972. The traffic assignment problem for multiclass-user transportation networks. Transportation Science 6, 73-87.

De Cea, J., Fernández, J.E., Dekock, V., Soto, A., 2005. Solving network equilibrium problems on multimodal urban transportation networks with multiple user classes. Transport Reviews 25, 293317.

Dempe, S., 2003. Annotated bibliography on bilevel programming and mathematical programs

This is the author manuscript accepted for publication and has undergone full peer review but has not been through the copyediting, typesetting, pagination and proofreading process, which may lead to differences between this version and the Version of Record. Please cite this article as doi: 10.1111/mice.12224.

This article is protected by copyright. All rights reserved. 
with equilibrium constraints. Optimization 52, 333359.

Drezner, Z., Wesolowsky, G.O., 1997. Selecting an optimum configuration of one-way and two-way routes. Transportation Science 31, 386-394.

Drezner, Z., Wesolowsky, G.O., 2003. Network design: selection and design of links and facility location. Transportation Research Part A: Policy and Practice 37, 241-256.

Estache, A., Ianchovichina, E., Bacon, R., Salamon, I., 2013. Infrastructure and employment creation in the Middle East and North Africa (MENA). Directions in development ; infrastructure. Washington D.C. : The Worldbank. 74918.

Farahani, R.Z., Miandoabchi, E., Szeto, W., Rashidi, H., 2013. A review of urban transportation network design problems. European Journal of Operational Research 229, 281-302.

Farvaresh, H., Sepehri, M.M., 2011. A single-level mixed integer linear formulation for a bi-level discrete network design problem. Transportation Research Part E: Logistics and Transportation Review 47, 623-640.

Farvaresh, H., Sepehri, M.M., 2013. A branch and bound algorithm for bi-level discrete network design problem. Netw Spat Econ 13, 67-106.

Ferguson, E.M., Duthie, J., Travis Waller, S., 2012. Comparing Delay Minimization and Emissions Minimization in the Network Design Problem. Computer-Aided Civil and Infrastructure Engineering 27, 288-302.

Florian, M., Morosan, C.D., 2014. On uniqueness and proportionality in multi-class equilibrium assignment. Transportation Research Part B: Methodological 70, 173-185.

Floudas, C.A., 1995. Nonlinear and mixed-integer optimization: fundamentals and applications. Oxford: Oxford University Press,.

Fontaine, P., Minner, S., 2014. Benders decomposition for discrete-continuous linear bilevel problems with application to traffic network design. Transportation Research Part B: Methodological 70, 163-172.

GAMS, 2014. GAMS Development Corporation, GAMS Development Corporation, Washington DC. .

Gao, Z., Wu, J., Sun, H., 2005. Solution algorithm for the bi-level discrete network design problem. Transportation Research Part B: Methodological 39, 479-495.

Geoffrion, A.M., 1972. Generalized Benders decomposition. Journal of Optimization Theory and Applications 10, 237-260.

INRO, 2009. EMME3 v 3.2, EMME3 User's Guide, 3.2 ed, Montreal, Quebec, Canada.

Larsson, T., Patriksson, M., 1995. An augmented Lagrangean dual algorithm for link capacity side constrained traffic assignment problems. Transportation Research Part B: Methodological 29, 433-455.
Lasdon, L.S., 2013. Optimization theory for large systems. Courier Corporation.

Leblanc, L.J., 1975. An Algorithm for the Discrete Network Design Problem. Transportation Science 9, 183-199.

Leyffer, S., 1993. Deterministic methods for mixed integer nonlinear programming. $\mathrm{PhD}$ University of Dundee.

Li, D., Sun, X., 2006. Nonlinear integer programming. Springer, Boston.

Lin, D.Y., 2011. A Dual Variable Approximation - Based Descent Method for a Bi level Continuous Dynamic Network Design Problem. Computer - Aided Civil and Infrastructure Engineering 26, 581-594.

Liu, Z., Meng, Q., 2012. Bus-based park-and-ride system: a stochastic model on multimodal network with congestion pricing schemes. International Journal of Systems Science 45, 994-1006.

Lo, H.K., Luo, X.W., Siu, B.W.Y., 2006. Degradable transport network: Travel time budget of travelers with heterogeneous risk aversion. Transportation Research Part B: Methodological 40, 792-806.

Lodi, A., 2010. Mixed integer programming computation, in: Jünger, M., Liebling, T.M., Naddef, D., Nemhauser, G.L., Pulleyblank, W.R., Reinelt, G., Rinaldi, G., Wolsey, L.A. (Eds.), 50 Years of Integer Programming 1958-2008. Springer Berlin Heidelberg, pp. 619-645.

Luathep, P., Sumalee, A., Lam, W.H.K., Li, Z.-C., Lo, H.K., 2011. Global optimization method for mixed transportation network design problem: A mixed-integer linear programming approach. Transportation Research Part B: Methodological 45, 808-827.

Magnanti, T.L., Wong, R.T., 1984. Network design and transportation planning: models and algorithms. Transportation Science 18, 1-55.

MathWorks, 2014. MATLAB and Statistics Toolbox, Release 2014a, , Natick, Massachusetts, United States.

Mesbah, M., Sarvi, M., Currie, G., 2011a. Optimization of Transit Priority in the Transportation Network Using a Genetic Algorithm. Intelligent Transportation Systems, IEEE Transactions on 12, 908-919.

Mesbah, M., Sarvi, M., Ouveysi, I., Currie, G., 2011b. Optimization of transit priority in the transportation network using a decomposition methodology. Transportation Research Part C: Emerging Technologies 19, 363-373.

Minoux, M., 1989. Networks synthesis and optimum network design problems: Models, solution methods and applications. Networks 19, 313-360.

Nagurney, A., 2000. A multiclass, multicriteria traffic network equilibrium model. Mathematical and Computer Modelling 32, 393-411.

Nagurney, A., Dong, J., 2002. A multiclass, multicriteria traffic network equilibrium model with 
elastic demand. Transportation Research Part B: Methodological 36, 445-469.

Newell, G.F., 1980. Traffic flow on transportation networks, MIT Press, Cambridge, Mass.

Nie, Y., Zhang, H., Lee, D.-H., 2004. Models and algorithms for the traffic assignment problem with link capacity constraints. Transportation Research Part B: Methodological 38, 285-312.

Patriksson, P., 1994. The traffic assignment problem: models and methods, VSP BV, The Netherlands. Facsimile reproduction published in 2014 by Dover Publications, Inc., Mineola, New York, NY, USA.

Poorzahedy, H., Turnquist, M.A., 1982. Approximate algorithms for the discrete network design problem. Transportation Research Part B: Methodological 16, 45-55.

Potts, R.B., Oliver, R.M., 1972. Flows in transportation networks, Academic Press, New York.

Roughgarden, T., Tardos, É., 2002. How bad is selfish routing? Journal of the ACM (JACM) 49, 236-259.

Ryu, S., Chen, A., Choi, K., 2015. Solving the stochastic multi-class traffic assignment problem with asymmetric interactions, route overlapping, and vehicle restrictions. Journal of Advanced Transportation, n/a-n/a.

Sahinidis, N., Grossmann, I.E., 1991. Convergence properties of generalized Benders decomposition. Computers \& chemical engineering 15, 481-491.

Sarvi, M., Kuwahara, M., 2008. Using ITS to improve the capacity of freeway merging sections by transferring freight vehicles. Intelligent Transportation Systems, IEEE Transactions on 9, 580-588

Sarvi, M., Kuwahara, M., Ceder, A., 2004. Freeway ramp merging phenomena in congested traffic using simulation combined with a driving simulator. Computer - Aided Civil and Infrastructure Engineering 19, 351-363.

Spiess, H., 1984. Contributions à la théorie et aux outils de planification des réseaux de transport urbain, $\mathrm{PhD}$ dissertation. Montréal: Université de Montréal, Centre de recherche sur les transports, Université de Montréal. Centre de recherche sur les transports.

Spiess, H., 1990. Technical note-Conical volumedelay functions. Transportation Science 24, 153-158.

Spiess, H., 1993. Transit equilibrium assignment based on optimal strategies: an implementation in EMME/2, EMME/2 Support Center, Haldenstrasse 16, $\mathrm{CH}-2558$ Aegerten, Switzerland.

Spiess, H., Florian, M., 1989. Optimal strategies: a new assignment model for transit networks. Transportation Research Part B: Methodological 23, 83-102.

Szeto, W.Y., Jaber, X., O’Mahony, M., 2010. Time-Dependent Discrete Network Design Frameworks Considering Land Use. Computer-
Aided Civil and Infrastructure Engineering 25, 411426.

Szeto, W.Y., Jiang, Y., Wang, D., Sumalee, A., 2013. A sustainable road network design problem with land use transportation interaction over time. Netw Spat Econ, 1-32.

Szeto, W.Y., Wang, A.B., 2015. Price of anarchy for reliability-based traffic assignment and network design. Transportmetrica A: Transport Science 11, 603-635.

Szeto, W.Y., Wang, Y., Wong, S.C., 2014. The Chemical Reaction Optimization Approach to Solving the Environmentally Sustainable Network Design Problem. Computer-Aided Civil and Infrastructure Engineering 29, 140-158.

Ukkusuri, S.V., Mathew, T.V., Waller, S.T., 2007. Robust Transportation Network Design Under Demand Uncertainty. Computer-Aided Civil and Infrastructure Engineering 22, 6-18.

Unnikrishnan, A., Lin, D.Y., 2012. User equilibrium with recourse: continuous network design problem. Computer - Aided Civil and Infrastructure Engineering 27, 512-524.

Waller, S.T., Mouskos, K.C., Kamaryiannis, D., Ziliaskopoulos, A.K., 2006. A linear model for the continuous network design problem. Computer Aided Civil and Infrastructure Engineering 21, 334345.

Wang, D.Z., Liu, H., Szeto, W., 2015. A novel discrete network design problem formulation and its global optimization solution algorithm. Transportation Research Part E: Logistics and Transportation Review 79, 213-230.

Wang, D.Z.W., Lo, H.K., 2010. Global optimum of the linearized network design problem with equilibrium flows. Transportation Research Part B: Methodological 44, 482-492.

Wang, S., Meng, Q., Yang, H., 2013. Global optimization methods for the discrete network design problem. Transportation Research Part B: Methodological 50, 42-60.

Wong, R.T., 1985. Transportation network research: Algorithmic and computational questions. Transportation Research Part A: General 19, 436438.

Xie, C., 2014. Bicriterion discrete equilibrium network design problem. Networks 63, 286-305.

Xie, J., Xie, C., 2014. An improved TAPAS algorithm for the traffic assignment problem, Intelligent Transportation Systems (ITSC), 2014 IEEE 17th International Conference on. IEEE, pp. 2336-2341.

Xie, J., Xie, C., 2015. Origin-Based Algorithms for Traffic Assignment: Algorithmic Structure, Complexity Analysis, and Convergence Performance, Transportation Research Board 94th Annual Meeting.

Yang, H., Bell, M.G.H., 1998. Models and algorithms for road network design: a review and 
some new developments. Transport Reviews 18, 257-278.

Zhang, G., Chen, J., 2010. Solving multi-class traffic assignment problem with genetic algorithm, Computational Intelligence and Natural Computing Proceedings (CINC), 2010 Second International Conference on. IEEE, pp. 229-232.

Zhang, H., Gao, Z., 2009. Bilevel programming model and solution method for mixed transportation network design problem. Journal of Systems Science and Complexity 22, 446-459.

Zheng, J.,Boyce, D., 2011. Comparison of UserEquilibrium and System-Optimal Route Flow Solutions under Increasing Traffic Congestion, Transportation Research Board 90th Annual Meeting.

\section{Appendix A: A single OD Example For the Benders decomposition algorithm}

Consider a network that consists of a single road (\#4) connecting an origin-destination pair with a travel demand of $q_{o d}=10$. There are three additional roads (\#1, \#2, \#3) designated to ease traffic but the budget can only afford a maximum of two roads $\left(c_{1}=c_{2}=c_{3}=1 ; B=2\right)$. Figure 2 depicts the problem as well as the delay functions associated with the roads. Initialization: consider $U=10, \varepsilon=0 \%$ and starting solution for binary variables $\left(y_{1}^{0}, y_{2}^{0}, y_{3}^{0}\right)=(0,0,0)$,

Iteration1: primal problem is formulated as follows (for the sake of brevity: subscript " $b "$, demand and conservative flow constraints are omitted):

$$
U B^{1}=\min \left\{x_{1}^{1}\left(.125 x_{1}^{1}+\omega_{1}^{1}\right)+x_{2}^{1}\left(.25 x_{2}^{1}+\omega_{2}^{1}\right)+x_{3}^{1}\left(.5 x_{3}^{1}+\omega_{3}^{1}\right)+x_{4}^{1} \cdot x_{4}^{1}\right\}
$$

The results of solving the above problem are: $\left(x_{1}^{1}, x_{2}^{1}, x_{3}^{1}, x_{4}^{1}\right)=(0,0,0,10) \quad, \quad\left(\omega_{1}^{1}, \omega_{2}^{1}, \omega_{3}^{1}\right)=(20,20,20) \quad$ and $U B=U B^{1}=100$. The relaxed master problem is formulated as:

$L B^{1}=\min \left\{V ;\right.$ s.t. $\left.V \geq 100-10 y_{1}^{1} 20-10 y_{2}^{1} 20-10 y_{3}^{1} 20 ; y_{1}^{1}+y_{2}^{1}+y_{3}^{1} \leq 2\right\}$

Solution found is $L B^{1}=-300,\left(y_{1}^{1}, y_{2}^{1}, y_{3}^{1}\right)=(1,0,1)$. Since $\left(U B^{*}-L B^{1}\right) / U B^{*}=100-(-300)>\varepsilon=0 \%$ the algorithm r proceeds to the next iteration. The objective functions of the primal problems along with the Benders cuts as well as numerical results for all the iterations are shown in Figure 2 and Table 5. The optimum solution is found at the end of the $2^{\text {nd }}$ iteration. But the algorithm continues further to make sure it is the global optimal solution by closing the gap between the upper and lower bounds.

\section{Appendix B: Merit index evaluations}

With respect to the problem presented in Appendix A which is a mixed integer quadratic programing problem, Figure 7 depicts a tableau of the problem solved by the $B \& B$ in two scenarios with and without the merit index ( $x_{i}$ is continuous and $y_{i}$ is binary variables). From the coefficient of the variables in the objective function, it is intuitively perceivable that the merit order of the binary variables is as $y_{1}, y_{2}, y_{3}$. The problem was solved using GAMS (GAMS, 2014), a leading optimization software. The optimal solution was found as follows:

$\left(y_{1}, y_{2}, y_{3}\right)=(1,1,0), \quad\left(x_{1}, x_{2}, x_{3}\right)=(6.1,3.1,0.0,0.8) \quad$ and $f(x, y)=7.7$. A quick comparison between the two scenarios highlights the significant and constructive role of the merit index in efficacy of the BB, such that the number of attempts to reach the global solution and total computational time increase almost three-fold should no merit index be considered. As noted before, the B\&B initiates on the candidate projects that are already sorted in order of their merit indices. Then, the algorithm takes y_a $=1$ branches on the tree as deep as possible because it is believed that the projects are wisely selected, hence the more we get of $y_{-} a=1$, the better we would be (this is also enforced by the budget consumption notion). Therefore, the proposed algorithm is a combination of the best-first-node and depth-first-node. The novelty and beauty of such selection rules is that the search becomes memoryless; that is, no need to keep track of the whole of the tree structure. 


\begin{tabular}{|c|c|}
\hline $\begin{array}{l}\text { Delay functions: } \quad \text { Demand: } q_{o d}=10 \\
\quad \text { Costs: } c_{1}=c_{2}=c_{3}=1 \\
t_{1}\left(x_{1}\right)=0.125 \cdot x_{1} \text { Budget: } B=2 \\
t_{2}\left(x_{2}\right)=0.25 \cdot x_{2} \\
t_{3}\left(x_{3}\right)=0.5 \cdot x_{3} \\
t_{4}\left(x_{4}\right)=1 \cdot x_{4}\end{array}$ & $\begin{array}{l}\text { Primal Problem: } \\
\text { Objective function in } 5 \text { successive iterations: } \\
U B^{1}=\min \left\{x_{1}^{1}\left(.125 x_{1}^{1}+\omega_{1}^{1}\right)+x_{2}^{1}\left(.25 x_{2}^{1}+\omega_{2}^{1}\right)+x_{3}^{1}\left(.5 x_{3}^{1}+\omega_{3}^{1}\right)+x_{4}^{1} \cdot x_{4}^{1}\right\} \\
U B^{2}=\min \left\{.125 x_{1}^{2} x_{1}^{2}+x_{2}^{2}\left(.25 x_{2}^{2}+\omega_{2}^{2}\right)+.5 x_{3}^{2} x_{3}^{2}+x_{4}^{2} \cdot x_{4}^{2}\right\} \\
U B^{3}=\min \left\{.125 x_{1}^{3} x_{1}^{3}+.25 x_{2}^{3} x_{2}^{3}+x_{3}^{2}\left(.5 x_{3}^{2}+\omega_{3}^{2}\right)+x_{4}^{2} \cdot x_{4}^{2}\right\} \\
U B^{4}=\min \left\{x_{1}^{4}\left(.125 x_{1}^{4}+\omega_{1}^{4}\right)+.25 x_{2}^{4} x_{2}^{4}+x_{3}^{4}\left(.5 x_{3}^{4}+\lambda_{3}^{4}\right)+x_{4}^{4} \cdot x_{4}^{4}\right\} \\
U B^{5}=\min \left\{x_{1}^{5}\left(.125 x_{1}^{5}+\omega_{1}^{5}\right)+.25 x_{2}^{5} x_{2}^{5}+.5 x_{3}^{5} x_{3}^{5}+x_{4}^{5} \cdot x_{4}^{5}\right\} \\
\text { Relaxed Problem: } \\
\text { Benders cuts as constraints accumulated in successive iterations } \\
V \geq 100-10 y_{1}^{1} 20-10 y_{2}^{1} 20-10 y_{3}^{1} 20 \\
V \geq 9.1-10 y_{2}^{2} 1.8 \\
V \geq 7.7-10 y_{3}^{3} 1.5 \\
V \geq 14.3-10 y_{1}^{5} 2.9\end{array}$ \\
\hline
\end{tabular}

Figure 6 a MINLP representing a single OD example solved by Benders algorithm

Table 5, single OD example: Iterative results of Benders algorithm

\begin{tabular}{|l|l|l|l|l|l|l|l|}
\hline $\mathrm{i}$ & $\mathrm{Y}^{\mathrm{i}-1}$ & $\mathrm{X}^{\mathrm{i}}$ & $\mathrm{W}^{\mathrm{i}}$ & $\mathrm{UB}^{\mathrm{i}}$ & $\mathrm{UB}^{1}$ & $\mathrm{LB}^{\mathrm{i}}$ & $\mathrm{Y}^{\mathrm{i}}$ \\
\hline 1 & $0,0,0$ & $0.0,0.0,0.0,10.0$ & $20.0,20.0,20.0$ & 100.0 & 100.0 & - & $1,0,1$ \\
\hline 2 & $1,0,1$ & $7.3,0.0,1.8,0.9$ & $0.0,1.8,0.0$ & 9.1 & 9.1 & -9.1 & $1,1,0$ \\
\hline 3 & $1,1,0$ & $6.1,3.1,0.0,0.8$ & $0.0,0.0,1.5$ & 7.7 & 7.7 & -7.7 & $0,1,1$ \\
\hline 4 & $0,1,1$ & $0.0,5.7,2.9,1.4$ & $2.9,0.0,0.0$ & 14.3 & 7.7 & 9.1 & $1,0,0$ \\
\hline
\end{tabular}

\section{Appendix C: Some Remarks \\ Remark 1, Efficacy of tree structure in the} lower bounds calculation: At each iteration a (parent) node renders two new (offspring) nodes, but it is only required to calculate the lower bound for one of the newly generated nodes. Because the other offspring node inherits the lower bound from the parent node, this makes the computation more efficient. Figure 13 depicts this observation graphically. The parent node corresponds to subarea "1122.22", has a lower bound corresponding to string "1110..01" with an objective function value of 85 . The branching is made at the third project (the very next project with value of " 2 ") which results in offspring nodes " $1112 . .22$ " in the left hand and " $1102 . .22$ " in the right hand. The third project in the string corresponding to the lower bound of the parent node is found " 1 ", so if we calculate the lower bound for the left hand offspring node (which has " 1 " at the third project), we will not find any solution better than that which has already been found. For the right hand node, the lower bound was found not better than the parent's lower bound $(88 \nless 85)$. If the lower bound string for parent node happened to be "1100..01" and the right hand offspring node were to inherit the lower bound from the parent, a new lower bound had to be calculated for the left hand node (Leblanc, 1975).

values are inherited through the tree

Remark 2, Benders algorithm; epsilon, convergence gap: In the proposed Benders algorithm, Step 3, epsilon $(\varepsilon)$ is a pre-specified parameter (in percentage) for which a perfect and desirable value is $0 \%$. Preliminary results of the application of Benders on large scale MINLP problems suggest that the global solution is likely to arrive at early iteration, while the epsilon only prolongs the computational time to close the gap between the upper and lower bounds. In order to speed up the algorithm, instead of the perfect value of 0 , we adopted a meagre value for epsilon (say $\varepsilon=0.2 \%$ ).

Remark 3, Benders algorithm; initial solution: In Step 0, the algorithm starts with an all-out null solution, that is $\left(y_{a}^{0}=0, \forall a \in A^{\prime}\right)$. One option is to look at the inventory of the best solutions found in the preceding node of the tree to see whether there is a binary solution that complies with the requirement of the current node. If nothing is found, then there still might be a better educated guess rather than the null scenario. One possibility is to fill the blank string from left to right with projects based on the merit index until the budget is depleted.

This technique has been implemented in the final computer code and was applied in the numerical analysis.

This is the author manuscript accepted for publication and has undergone full peer review but has not been through the copyediting, typesetting, pagination and proofreading process, which may lead to differences between this version and the Version of Record. Please cite this article as doi: $10.1111 /$ mice. 12224 .

This article is protected by copyright. All rights reserved. 
Remark 4, Benders algorithm; a greedy search for better incumbent value (I): Benders algorithm is devised to render a tight lower bound based on the MMMC-SO traffic flow. The corresponding binary solution of the best solution emerged out of the Benders algorithm and might render a much better incumbent value too. As a result, at the end of each Benders algorithm the resulted binary solution is taken and for which MMMC-UE-TAP is solved too. The outcome is used to update the incumbent value. The rationale behind the adjustment of the algorithm is as follows: the SO version of the network design problem is a good approximation for the UE version. It is important to note that the first lower bound is calculated for the root node of the B\&B's tree (where representing the entire solution space by string " $22 . .22$ ". In fact, this lower bound is equivalent to solving a SO version of the NDP which is proven to be easier and this is called SO-relaxation (Wang et al., 2013). This notion is strongly reinforced in the numerical analysis (next section) such that in most cases, the global optimal solution is achieved in the first lower bound calculated at the root node.

This technique has been implemented in the final computer code and was applied in the numerical analysis.

Remark 5, Benders algorithm; a greedy search for better incumbent value (II): Provided the candidate projects have been selected wisely, a good solution is expected to consume the budget to its full. The more projects that contribute to the solution, the better the solution becomes. To this end, to force more of value" 1 " in the binary strings $\left(y_{a}\right)$, the objective function of the Relaxed Master Problem (equation (20)) is changed to: $V-\sum_{a \in A^{\prime}} y_{a}$. The newly added second term $\left(-\sum_{a \in A^{\prime}} y_{a}\right)$ is called "budget consumption term". In the next section (numerical tests), we report on both cases with and without the budget consumption term.

Remark 6, A much tighter System Optimal lower bound: No matter how perfect the attempt is to find the maximum possible lower bound, since the lower bound is based on the System Optimal (SO) traffic flow, the gap between SO lower bound and UE incumbent value might be very significant by nature. Roughgarden and Tardos (2002) mathematically proved that the UE incumbent value can be as high as 2.15 times the SO lower bound in networks governed by BPR delay functions (Recently, a similar result has been reported by (Szeto and Wang, 2015). In the following discussion, we first highlight the causes of the aforementioned gap. We then propose a arameter to close this deep gap.

The SO flow can be easily computed (even using commercial transport planning software) by replacing $t_{a}\left(x_{a}+\bar{x}_{a}\right)$ the delay function in the
Beckmann objective function of the UE flow (equation (4)) to $\tilde{t}_{a}\left(x_{a}+\bar{x}_{a}\right)$ (Newell, 1980; Potts and Oliver, 1972):

$$
\tilde{t}_{a}\left(x_{a}+\bar{x}_{a}\right)=t_{a}\left(x_{a}+\bar{x}_{a}\right)+x_{a} \cdot \partial t_{a}\left(x_{a}+\bar{x}_{a}\right) / \partial x_{a}
$$

If $t_{a}\left(x_{a}+\bar{x}_{a}\right)$ is considered as the cost of traveling on a road $a \in A$, then $\tilde{t}_{a}\left(x_{a}+\bar{x}_{a}\right)$ is known as the marginal cost of using the respective road. The deep gap between SO and UE emerges from the second term in the right side of the equation (24) which is the additional externality cost imposed to the users. The two functions $t, \tilde{t}$ show benign and similar behaviour as long as the volume is below capacity. As the volume gets close to or exceeds capacity, the externality cost increases rapidly, which results in the deep gap between the SO and UE flows. Since the delay functions are not capacity restricted, in theory, the volume and hence the delay as well as the marginal cost can increase to infinity. This results in a much deeper gap between SO and UE. In order to close such an unrealistic gap, we propose alpha $0 \leq \alpha \leq 1$ a coefficient to the externality term as follows:

$\tilde{t}_{a}\left(x_{a}+\bar{x}_{a}\right)=t_{a}\left(x_{a}+\bar{x}_{a}\right)+\alpha \cdot x_{a} \cdot \partial t_{a}\left(x_{a}+\bar{x}_{a}\right) / \partial x_{a}$ 


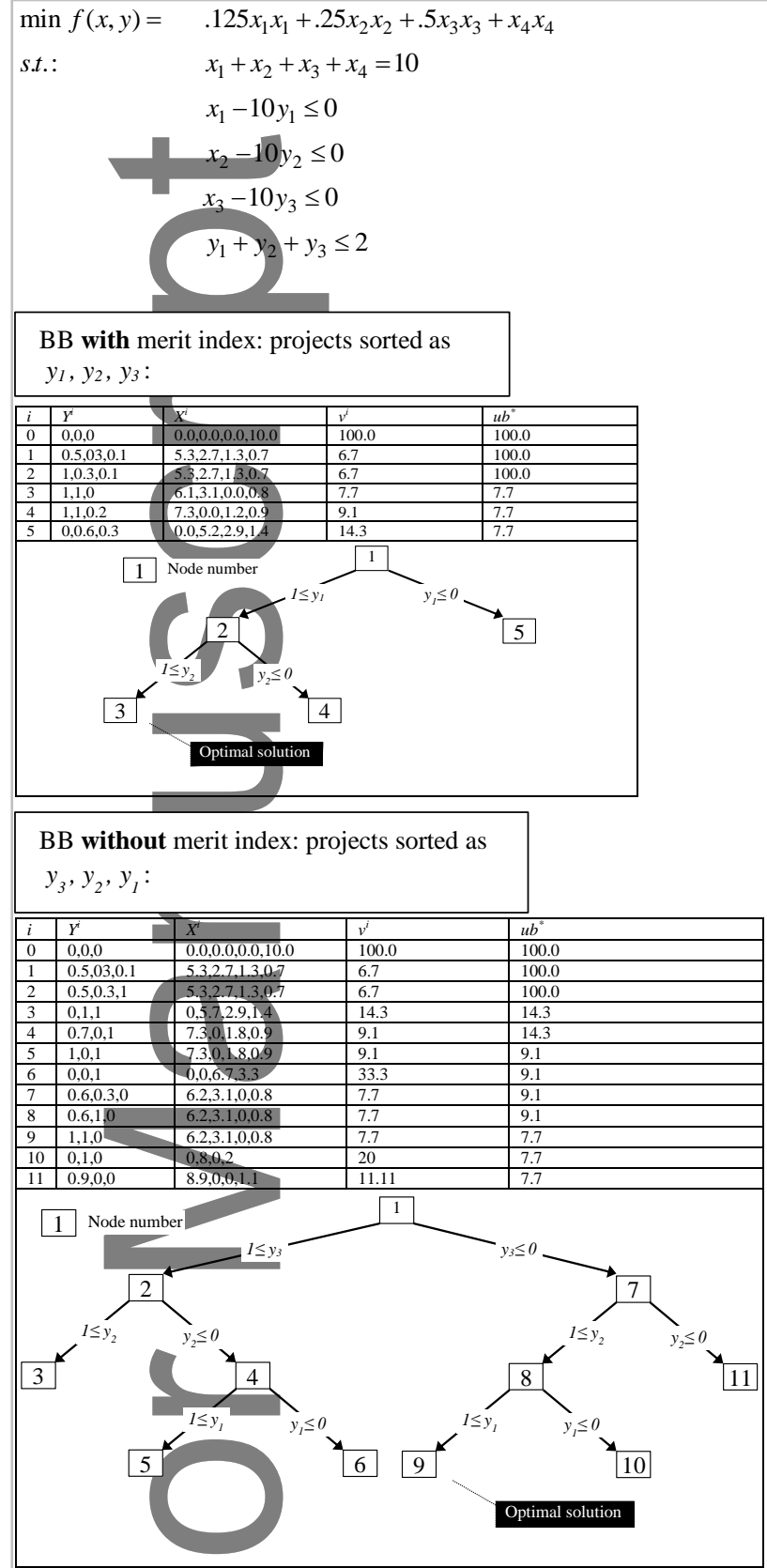

FIGURE 7 Branch-and-Bound performance with and without the merit index (Note: $v^{i}$ is value of the objective function at iteration $i$, and $u b^{*}$ is the best upper bound or the incumbent value)

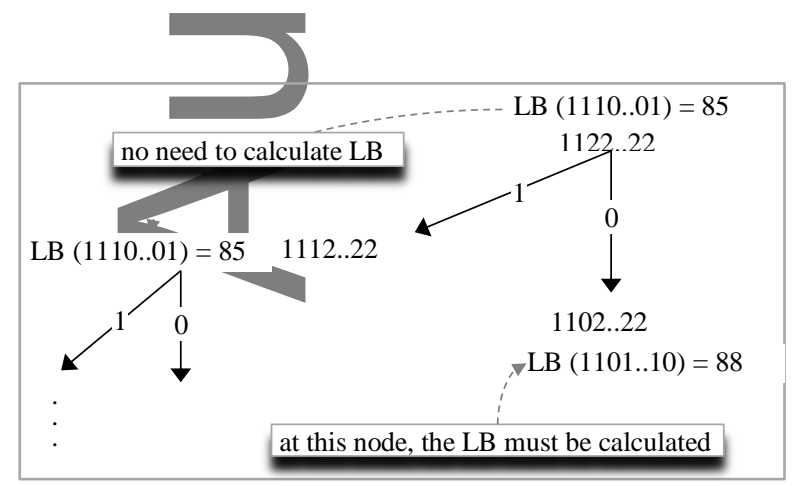

Figure 8 Illustration of how the lower bound
As alpha gets close to zero, the SO gets close to $\mathrm{UE}$ and the gap vanishes. It is worth noting that the alpha addresses the unfortunate trade-off between the computational time (CPU time) and accuracy of the final results. Lower values of alpha lead to faster but less accurate results. As such, the value of alpha can be set as per the user's discretion depending on the computational technology at the time and how affordable the computational time is. In other words, alpha is a value in the hand of the modeller based on which the accuracy of the results along with the computational time can be adjusted depending on the size of the network, the available computational technology and the strategic value of the final solution. In addition, adopting any positive value below 1 for alpha is a diversion from solving a full SO to semi SO network design problem ${ }^{16}$

The numerical results suggest that even a trivial value of alpha (say $\alpha=0.005$ ) is enough to secure global optimal solutions (which were already identified through an exhaustive enumeration). Nevertheless, the variation of results over the variation of alpha is a question that remains to be further studied $^{17}$.

${ }^{16}$ As mentioned early, according to the work of Roughgarden, T., Tardos, É., 2002. How bad is selfish routing? Journal of the ACM (JACM) 49, 236-259. the gap between SO and UE can be as high as 2.15 times. A recent review on this subject is provided by Wang, D.Z., Liu, H., Szeto, W., 2015. A novel discrete network design problem formulation and its global optimization solution algorithm. Transportation Research Part E: Logistics and Transportation Review 79, 213-230. . Though 2.15 is proven to be an upper bound for typical road networks, the works of Prof Boyce (Boyce, D., Xiong, Q., 2004. Useroptimal and system-optimal route choices for a large road network. Review of network Economics 3, Zheng, J., Boyce, D., 2011. Comparison of User-Equilibrium and System-Optimal Route Flow Solutions under Increasing Traffic Congestion, Transportation Research Board 90th Annual Meeting.) suggest that the 2.15 is perhaps a very loose upper bound for real-life networks such as Chicago. Therefore it opens up a decent avenue for further investigation. Nevertheless, the gap between SO and UE is exactly the area at which the congestion pricing emerges in real world. We all know that congestion pricing is still hotly debated. Problem (11) is devised to find a SO based lower bound value. According to Remark 6, in order to find a tighter lower bound value term alpha is proposed. According to equation (24), the alpha (which varies between 0 and 1) is applied to the derivative term of the travel time, to dampen the gap between normal travel time (of UE) and marginal travel time (of SO). Problem (11) subject to alpha equals to 1 becomes the lower bound problem as suggested and proven by Leblanc (1975). As we discussed in Remark 6, although any other (positive) value of alpha (less than 1) gets a lower bound value higher than the valid value (pertaining to alpha=1), it helps to speed up the algorithm. The numerical results of Winnpeg suggests that even a trivial value of alpha is enough to guarantee the global optimum solution in tolerable CPU time.

${ }^{17}$ The efficacy of any Branch-and-Bound algorithm is tied to whether the algorithm can efficiently found and discard inferior subareas, that is, the subarea of the solution space at which the global optimum solution certainly doesn't exist. To do so, the SO lower bound value must be found higher that the incumbent value (UE upper bound). Normally, the SO lower bound is way below the UE upper bound. The alpha is then purposely devised to artificially lift the SO lower bound up close to the UE upper bound aiming at increasing the likelihood of having the SO 
Remark 7, A note on convergence of the Benders algorithm; In some circumstances Generalized Benders Decomposition does not converge when the NLP is non-convex, (Bagajewicz and Manousiouthakis, 1991). In the following exposition, we elaborate on these circumstances and discuss why our proposed algorithm does not fall into such circumstances.

The important point to note is that given a feasible solution of binary variables (y), problem (11) is convex on the continuous variables of the roads' traffic volume (x). In fact, for every feasible solution y, problem (11) is a system-optimal traffic assignment problem which has a guaranteed unique solution. According to(Bagajewicz and Manousiouthakis, 1991), the key to avoid stagnation in the local optimum is a condition coined by Geoffrion (1972) known as "property (P)". Property $\mathrm{p}$ stands for the situation at which the lagrangian relaxation problem can be taken, essentially independently of $\mathrm{y}$, so that the dual problem (equation (20)..(23)) can be solved on $y$. This is exactly what is being conducted in solving the lower bound problem. Generally speaking, convergence of the scheme is guaranteed if whenever the integer values $\mathrm{y}$ are fixed to some feasible vector - the remaining continuous subproblem is convex and is such that strong duality holds (the Lagrangian dual maximum value is equal to the primal optimum value)(Sahinidis and Grossmann, 1991).

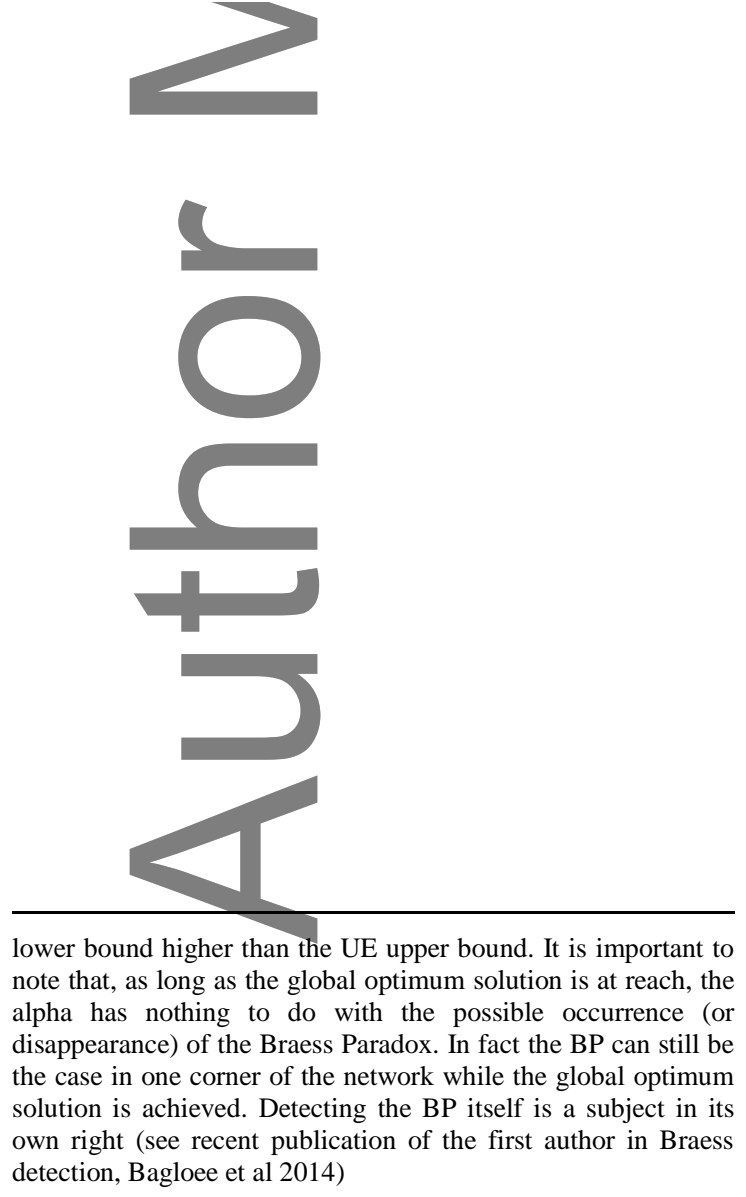




\section{University Library}

\section{- M M N E R VA A gateway to Melbourne's research publications}

Minerva Access is the Institutional Repository of The University of Melbourne

Author/s:

Bagloee, SA;Sarvi, M;Patriksson, M

Title:

A Hybrid Branch-and-Bound and Benders Decomposition Algorithm for the Network Design Problem

Date:

2017-04-01

Citation:

Bagloee, S. A., Sarvi, M. \& Patriksson, M. (2017). A Hybrid Branch-and-Bound and Benders Decomposition Algorithm for the Network Design Problem. COMPUTER-AIDED CIVIL AND INFRASTRUCTURE ENGINEERING, 32 (4), pp.319-343. https://doi.org/10.1111/mice.12224.

Persistent Link:

http://hdl.handle.net/11343/291723 